Pure and Applied Mathematics Quarterly

Volume 5, Number 2

(Special Issue: In honor of

Friedrich Hirzebruch, Part 1 of 2)

641-700, 2009

\title{
Birational Geometry of Algebraic Varieties with a Pencil of Fano Cyclic Covers
}

\author{
A.V. Pukhlikov \\ To Professor Friedrich Hirzebruch on the occasion of his eightieth birthday
}

\begin{abstract}
We prove birational rigidity of fiber spaces $\pi: V \rightarrow \mathbb{P}^{1}$, the fiber of which is a Fano cyclic cover of index 1, provided it is sufficiently twisted over the base. In particular, non-rationality of these varieties is shown and their group of birational automorphisms is computed. The proof is obtained by a combination of the classical quadratic technique of the method of maximal singularities with the technique of hypertangent divisors and the connectedness principle of Shokurov and Kollár.
\end{abstract}

Bibliography: 43 titles.

Keywords: birational rigidity, birational self-map, rationality, linear system, singularity.

Introduction

0.1. Birational classification of algebraic varieties

0.2. Rationally connected fiber spaces and the problem of birational rigidity

0.3. Fano cyclic covers

0.4. The regularity conditions

0.5. Formulation of the main result

Received August 7, 2006. 
0.6. The structure of the proof

0.7. Historical remarks

1. The method of maximal singularities

1.1. Start of the proof of Theorem 4

1.2. The structure of the sequence of blow ups

1.3. Multiplicities of the horizontal cycles

1.4. The technique of counting multiplicities

1.5. The supermaximal singularity

2. The technique of hypertangent divisors

2.1. Hypertangent divisors

2.2. Hypertangent linear systems

2.3. Checking the condition $(f)$

3. Exclusion of maximal singularities lying over a double point of a fiber

3.1. The singular point outside the ramification divisor

3.2. The singular point on the ramification divisor

3.3. Degrees and multiplicities

4. Explicit constructions

4.1. An explicit construction of Fano fiber spaces

4.2. Varieties of type $((0),(2,0))$

4.3. Varieties of type $((1,1),(0,0))$

4.4. Varieties with a unique structure of a fiber space

4.5. Non-rigid varieties

4.5.1. Varieties of type $((0),(0,0))$ 
5. Proof of the regularity conditions

5.1. Singularities of fibers

5.2. Existence of regular varieties

5.3. An estimate for the codimension

References

\section{INTRODUCTION}

0.1. Birational classification of algebraic varieties. One of the main tasks of algebraic geometry is classification of algebraic varieties. However, classification up to an isomorphism makes sense inside certain special classes of varieties only (except for the curves), since algebraic varieties are "too numerous" and already for surfaces the biregular classification is non-observable. Starting at the dimension two, it is birational geometry that gives a correct approach to the task of classification. Birational geometry does not make difference between birationally equivalent models of the same function field. More exactly, birational geometry makes it possible to specify for a given field of rational functions a compact set of models that admit a natural geometric description and are convenient to work with. One of the most important classification tools is reducing to a smaller dimension. If an algebraic variety $V$ admits a morphism $\pi: V \rightarrow S$ onto a variety $S$ of positive dimension, where $\operatorname{dim} S<\operatorname{dim} V$, then it is natural to consider $V$ as a family of varieties of dimension $\operatorname{dim} V-\operatorname{dim} S$ (that is, the fibers $F_{s}=\pi^{-1}(s)$, $s \in S$ ), parameterized by the base $S$. Thus description of the variety $V$ is reduced to description of geometry of the fiber of general position $F_{s}$ and of the "twistedness" of the family $F_{s}, s \in S$, over the base $S$.

Fiber spaces $\pi: V \rightarrow S$ can be considered from the two viewpoints. Firstly, fixing a class $\mathcal{S}$ of varieties $S$ and a class $\mathcal{F}$ of varieties $F_{s}$, one can construct new varieties of $\operatorname{dimension} \operatorname{dim} S+\operatorname{dim} F$ as fiber spaces with a given base $S \in \mathcal{S}$ and fibers $F_{s} \in \mathcal{F}$. Thus we get a method of constructing new algebraic varieties of arbitrary dimension. Secondly, for a given variety $X$ one may ask: does there exist 
a birational equivalence

$$
\begin{array}{r}
X \stackrel{\chi}{\rightarrow} \text { V } \\
\downarrow \\
S,
\end{array}
$$

where $V / S$ is a fiber space with the base and fibers taken from fixed classes $\mathcal{S}$ and $\mathcal{F}$, respectively? In other words, does there exist a rational map

$$
\rho(=\pi \circ \chi): X \rightarrow S,
$$

$S \in \mathcal{S}$, the fiber of which $\rho^{-1}(s)$ is birational to a variety from the given class $\mathcal{F}$ ?

These informal arguments obviously imply that those varieties are of exceptional importance in geometry which cannot be fibered by a rational map into varieties of a given type, or can be fibered in a unique way. Such varieties do not permit a reduction of dimension (or permit it in a unique way) and for this reason represent a new classification type. One can say that these varieties "do not come from smaller dimensions". In the next section we will give a precise meaning to this informal discussion of fiber spaces, fixing the class of the fiber and of the base, and after that we formulate the problem of birational rigidity. This problem deals with varieties which can be represented as fiber spaces (of a certain natural type) in finitely many ways.

0.2. Rationally connected fiber spaces and the problem of birational rigidity. The modern birational geometry started with the negative solution of the Lüroth problem $[2,5,15]$. Since unirational varieties have no non-zero global sections of covariant tensor bundles, the negative solution to the Lüroth problem meant that there was no hope to characterize higher-dimensional rational varieties in terms of differential geometry, similar to dimension one (the Lüroth theorem) and two (the Castelnuovo rationality criterion). The class of varieties with trivial differential-geometric invariants turned out to be much bigger than that of rational varieties. Due to the papers of Kollár, Miyaoka, Mori [17,18] and Graber, Harris, Starr [9] it is clear now, that the place in the general classification picture, which in low dimensions is occupied by rational curves and surfaces, in dimension three and higher belongs to rationally connected varieties. By definition $[9,17,18]$, a variety $X$ is said to be rationally connected, if any two points of general position $x, y \in X$ can be joined by an irreducible rational curve. (For instance, any two points $x, y \in \mathbb{P}^{n}$ lie on a line.) It is easy to see that the image of a rationally 
connected variety with respect to any rational map is rationally connected. In [9] it is proven that if the image of the variety $X$ and the fiber of general position are rationally connected, then the variety $X$ is rationally connected, either. If $\pi: V \rightarrow S$ is a morphism with connected fibers, and moreover the base $S$ and the general fiber $F_{s}=\pi^{-1}(s), s \in S$, are rationally connected, we call the fiber space $V / S$ a rationally connected fiber space. Thus the theorem of Graber-Harris-Starr [9] asserts that if $V / S$ is a rationally connected fiber space, then $V$ is a rationally connected variety.

A rationally connected variety $V$ has trivial differential-geometric invariants; in particular, all pluricanonical linear systems are empty: $\left|m K_{V}\right|=\emptyset, m \geq 1$. However, we can say more: the canonical class $K_{V}$ is negative on a dense family of rational curves, sweeping out $V$. Thus a rationally connected variety satisfies the classical condition of termination of canonical adjunction, that is, for any divisor $D$ the linear systems $\left|D+m K_{V}\right|$ are empty for $m \geq m(D)$. The condition of termination of adjunction played an important part in the classical geometry, for instance, in the proof of the Castelnuovo rationality criterion, see [1,14] for a modern exposition. To formalize this condition in a more precise way, recall some standard definitions. For a smooth rationally connected variety $X$ consider the Chow group $A^{i} X$ of algebraic cycles of codimension $i$ modulo numerical equivalence, $A^{1} X \cong \operatorname{Pic} X$, and set $A_{\mathbb{R}}^{i} X=A^{i} X \otimes \mathbb{R}$. Let

$$
A_{+}^{i} X \subset A_{\mathbb{R}}^{i} X
$$

be the closed cone, generated by effective classes, that is, the cone of pseudoeffective cycles. Set also

$$
A_{\mathrm{mov}}^{1} X \subset A_{\mathbb{R}}^{1} X
$$

to be the closed cone, generated by the classes of generic divisors of movable linear systems.

Definition 0.1. (i) The threshold of canonical adjunction of the divisor $D$ on the variety $X$ is the number

$$
c(D, X)=\sup \left\{\varepsilon \in \mathbb{Q}_{+} \mid D+\varepsilon K_{X} \in A_{+}^{1} X\right\} .
$$

If $\Sigma$ is a non-empty linear system on $X$, then we set $c(\Sigma, X)=c(D, X)$, where $D \in \Sigma$ is some divisor. 
(ii) For a movable linear system $\Sigma$ on the variety $X$ define the virtual threshold of canonical adjunction by the formula

$$
c_{\mathrm{virt}}(\Sigma)=\inf _{X^{\sharp} \rightarrow X}\left\{c\left(\Sigma^{\sharp}, X^{\sharp}\right)\right\},
$$

where the infimum is taken over all birational morphisms $X^{\sharp} \rightarrow X, X^{\sharp}$ is a smooth projective model of the field $\mathbb{C}(X), \Sigma^{\sharp}$ is the strict transform of the system $\Sigma$ on $X^{\sharp}$.

As a rule, it is easy to compute the threshold of canonical adjunction; for this reason, it is not hard to describe movable linear systems with a given value of the threshold of canonical adjunction. On the contrary, the virtual threshold of canonical adjunction is a very non-trivial invariant, since its definition involves the operation of taking the infimum over all models. The importance of the virtual threshold comes from the fact that it is obviously a birational invariant of the pair $(V, \Sigma)$

The following claim is almost obvious.

Proposition 0.1. Let $\pi: V \rightarrow S$ be a rationally connected fiber space, $\Lambda$ a movable system on $S, \operatorname{dim} S \geq 1$. Then $c\left(V, \pi^{*} \Lambda\right)=0$.

Proof. Any divisor $D \in \pi^{*} \Lambda$ is trivial on the fibers of $\pi$, whereas the canonical class $K_{V}$ is negative on a dense family of rational curves sweeping out the fiber of general position. Q.E.D. for the proposition.

Corollary 0.1. (i) Assume that on the variety $V$ there are no linear systems with the zero virtual threshold of canonical adjunction. Then on $V$ there are no structures of a non-trivial fiber space into varieties of negative Kodaira dimension, that is, there is no rational dominant map $\rho: V \rightarrow S$, $\operatorname{dim} S \geq 1$, the fiber of general position of which has the negative Kodaira dimension.

(ii) Let $\pi: V \rightarrow S$ be a rationally connected fiber space. Assume that every movable linear system $\Sigma$ on $V$ with the zero virtual threshold of canonical adjunction, $c_{\mathrm{virt}}(\Sigma)=0$, is a pull back from the base: $\Sigma=\pi^{*} \Lambda$, where $\Lambda$ is some movable linear system on $S$. Then every birational map

$$
\begin{aligned}
& V_{-\stackrel{\chi}{\rightarrow}}^{\rightarrow} V^{\sharp} \\
& \pi \downarrow \quad \downarrow \pi^{\sharp} \\
& S \quad S^{\sharp} \text {, }
\end{aligned}
$$


where $\pi^{\sharp}: V^{\sharp} \rightarrow S^{\sharp}$ is a fiber space into varieties of negative Kodaira dimension, is fiber-wise, that is, there exists a rational dominant map $\rho: S \rightarrow S^{\sharp}$, making the diagram (1) commutative, $\pi^{\sharp} \circ \chi=\rho \circ \pi$.

Corollary 0.1 shows that the virtual thresholds of canonical adjunction make it possible to give a complete description of birational geometry of a variety. However, their computation is a very non-trivial task. The ordinary thresholds of canonical adjunction are easy to compute, but they do not give much from the birational viewpoint. These arguments show the importance of the following

Definition 0.2. (i) A variety $V$ is said to be birationally superrigid, if for every movable linear system $\Sigma$ on $V$ the equality

$$
c_{\mathrm{virt}}(\Sigma)=c(\Sigma, V)
$$

holds.

(ii) A variety $V$ (respectively, a Fano fiber space $V / S$ ) is said to be birationally rigid, if for every movable linear system $\Sigma$ on $V$ there exists a birational selfmap $\chi \in \operatorname{Bir} V$ (respectively, a fiber-wise self-map $\chi \in \operatorname{Bir}(V / S)$ ), producing the equality

$$
c_{\mathrm{virt}}(\Sigma)=c\left(\chi_{*} \Sigma, V\right)
$$

The property of being birationally (super)rigid reduces computation of the virtual threshold of canonical adjunction to the computation of the ordinary threshold of canonical adjunction, after which the problem of description of non-trivial structures of a fibration into varieties of negative Kodaira dimension becomes accessible, often very simple (and other problems of birational geometry for the given variety, as well). Moreover, today there are no other methods of description of the structures of a fiber space, except for by solving the problem of birational rigidity. For this reason, the problem of birational rigidity is of fundamental importance.

The aim of this paper is to solve the problem of birational rigidity for Fano cyclic covers of index one and varieties with a pencil of Fano cyclic covers.

0.3. Fano cyclic covers. Set $\mathbb{P}=\mathbb{P}^{M+1}$ to be the complex projective space, $M \geq 5, G=G_{m} \subset \mathbb{P}$ to be an irreducible hypersurface of degree $m \geq 2, \sigma: F \rightarrow G$ to be the cyclic cover of degree $K \geq 3$, branched over an irreducible divisor $W \cap G$, where $W=W_{K l} \subset \mathbb{P}$ is a hypersurface of degree $K l$. Introducing a new coordinate $u$ of weight $l$, one can realize $F$ as a complete intersection of the type $m \cdot K l$ in 
the weighted projective space

$$
\mathbb{P}(\underbrace{1, \ldots, 1}_{M+2}, l) .
$$

Namely, $F$ is given by the system of equations

$$
\left\{\begin{array}{l}
f\left(x_{0}, \ldots, x_{M+1}\right)=0 \\
u^{K}=g\left(x_{0}, \ldots, x_{M+1}\right)
\end{array}\right.
$$

where $f\left(x_{*}\right)$ and $g\left(x_{*}\right)$ are homogeneous polynomials of degrees $m$ and $K l$, respectively.

If the integers $m, l$ and $K$ satisfy the relation $m+(K-1) l=M+1$, then $F$ is a primitive Fano variety of dimension $M$, that is, $\operatorname{Pic} F=\mathbb{Z} K_{F}$ and $\left(-K_{F}\right)$ is ample .

The first result of the present paper is the following

Theorem 1. A sufficiently general (in the sense of Zariski topology) variety $F$ is birationally superrigid.

In particular, $F$ admits no non-trivial structures of a fibration into varieties of negative Kodaira dimension, every birational map $F \rightarrow F^{\sharp}$ onto a Fano variety $F^{\sharp}$ with $\mathbb{Q}$-factorial terminal singularities and $\operatorname{rkPic} F^{\sharp}=1$ is an isomorphism and the groups of birational and biregular automorphisms coincide: $\operatorname{Bir} F=\operatorname{Aut} F$.

Remark 0.1. The claim of Theorem 1 can be made somewhat stronger: for the variety $F^{\sharp}$ one can take any Fano variety with canonical singularities, and apart from that, it is easy to describe all structures of a fibration on $F$, the relative Kodaira dimension of which is strictly less than the maximal one. For the standard procedure of such a generalization, see [34,40-42].

The main object of study in this paper are varieties with a pencil of Fano cyclic covers. From this moment on, we assume that the integers $m, K, l$ are fixed and consider the open set

$$
\mathcal{F} \subset \mathbb{P}\left(H^{0}\left(\mathbb{P}, \mathcal{O}_{\mathbb{P}}(m)\right)\right) \times \mathbb{P}\left(H^{0}\left(\mathbb{P}, \mathcal{O}_{\mathbb{P}}(K l)\right)\right),
$$

defined by the following condition: for a pair of polynomials $(f, g) \in \mathcal{F}$ the corresponding Fano cyclic cover $F$ is irreducible. The set $\mathcal{F}$ makes a natural parameter space for Fano cyclic covers. 
A variety with a pencil of Fano cyclic covers is a smooth fiber space $\pi: V \rightarrow \mathbb{P}^{1}$, each fiber of which is a Fano cyclic cover, that is, a variety from the family $\mathcal{F}$. The aim of this paper is to prove that "almost all" (in a certain natural sense that will be explained below) such fiber spaces $V / \mathbb{P}^{1}$ are birationally superrigid, in particular, they have a unique non-trivial structure of a fibration into varieties of negative Kodaira dimension, that is, the projection $\pi: V \rightarrow \mathbb{P}^{1}$.

In order to give a precise formulation of the main result, we need some local conditions of general position (regularity) for the varieties $F \in \mathcal{F}$.

0.4. The regularity conditions. The set of smooth cyclic covers $F \in \mathcal{F}$ we denote by the symbol $\mathcal{F}_{\text {sm }}$, its complement $\mathcal{F} \backslash \mathcal{F}_{\text {sm }}$ (that is, the set of singular cyclic covers) by the symbol $\mathcal{F}_{\text {sing }}$.

Let $F \sim(f, g) \in \mathcal{F}$ be a variety, $o \in F$ an arbitrary point, $p=\sigma(o) \in G=\{f=$ $0\}$ its image on $\mathbb{P}$. Choose a system of affine coordinates $z_{1}, \ldots, z_{M+1}$ with the origin at the point $p$. Without loss of generality we may assume that $z_{i}=x_{i} / x_{0}$. Set $y=u / x_{0}^{l}$. Now the standard affine set

$$
\mathbb{A}_{\left(z_{1}, \ldots, z_{M+1}, y\right)}^{M+2}
$$

is a chart for $\mathbb{P}(1, \ldots, 1, l)$. Abusing our notations, we use for the non-homogeneous polynomials, corresponding to $f$ and $g$, the same symbols:

$$
f=q_{1}+\cdots+q_{m}, \quad g=w_{0}+w_{1}+\cdots+w_{K l},
$$

where $q_{i}, w_{j}$ are homogeneous components of degree $i, j$ in the variables $z_{*}$, respectively, so that in the affine chart $\mathbb{A}_{\left(z_{*}, y\right)}^{M+2}$, introduced above, the variety $F$ is given by the pair of equations $f=0, y^{K}=g$ (replacing the system (2)). If the point $o \in F$ does not lie on the ramification divisor of the morphism $\sigma$, then we always assume that $w_{0}=1$. If the point $p \in G$ is non-singular, then without loss of generality we assume that $q_{1} \equiv z_{M+1}$. In the latter case we set

$$
\bar{q}_{i}=\left.q_{i}\right|_{\left\{z_{M+1}=0\right\}}=q_{i}\left(z_{1}, \ldots, z_{M}, 0\right)
$$

and $\bar{w}_{j}=\left.w_{j}\right|_{z_{M+1}=0}=w_{j}\left(z_{1}, \ldots, z_{M}, 0\right)$ for $i, j \geq 2$.

Now let us formulate the regularity condition for a point $o \in F$. We consider separately the four cases, depending on the point $o \in F$ being smooth or singular, and on whether it lies on the ramification divisor or not. 
(R1.1) The regularity condition for a smooth point outside the ramification divisor. Here $w_{0}=1$ and we may assume that $y(o)=1$. Similar to [22,28], set

$$
\begin{gathered}
g^{1 / K}=\left(1+w_{1}+\cdots+w_{K l}\right)^{1 / K}=1+\sum_{i=1}^{\infty} \gamma_{i}\left(w_{1}+\cdots+w_{K l}\right)^{i}= \\
=1+\sum_{i=1}^{\infty} \Phi_{i}\left(w_{1}, \ldots, w_{K l}\right)
\end{gathered}
$$

where $\gamma_{i} \in \mathbb{Q}$ are defined by the Taylor series of the function $(1+s)^{1 / K}$ at zero, $s$ is a variable:

$$
(1+s)^{1 / K}=1+\sum_{i=1}^{\infty} \gamma_{i} s^{i}
$$

and $\Phi_{i}\left(w_{1}\left(z_{*}\right), \ldots, w_{K l}\left(z_{*}\right)\right)$ are homogeneous polynomials of degree $i \geq 1$ in the variables $z_{*}$. It is easy to see that for $i \in\{1, \ldots, K l\}$ we get

$$
\Phi_{i}\left(w_{*}\left(z_{*}\right)\right)=\frac{1}{K} w_{i}+\Phi_{i}^{\sharp}\left(w_{1}, \ldots, w_{i-1}\right) .
$$

In these notations the regularity condition is formulated in the following way: for $m \leq K l$ the sequence

$$
q_{1}, \ldots, q_{m}, \Phi_{l+1}\left(w_{*}\left(z_{*}\right)\right), \ldots, \Phi_{K l-1}\left(w_{*}\left(z_{*}\right)\right)
$$

should be regular in $\mathcal{O}_{o, \mathbb{C}^{M+1}}$; if $m \geq K l+1$, then we require the regularity of the following sequence:

$$
q_{1}, \ldots, q_{m-1}, \Phi_{l+1}\left(w_{*}\left(z_{*}\right)\right), \ldots, \Phi_{K l}\left(w_{*}\left(z_{*}\right)\right) .
$$

(R2.1) The regularity condition for a smooth point on the ramification divisor. Here $w_{0}=0$. We require that the sequence

$$
q_{1}, \ldots, q_{m}, w_{1}, \ldots, w_{K}
$$

were regular in $\mathcal{O}_{o, \mathbb{C}^{M+1}}$.

(R1.2) The regularity condition for a singular point outside the ramification divisor. This condition is similar to the condition (R1.1). Here $w_{0}=1$, $y(o)=1$ and in the notations of the condition (R1.1) we require that the sequence of homogeneous polynomials

$$
q_{2}, \ldots, q_{m}, \Phi_{l+1}\left(w_{*}\left(z_{*}\right)\right), \ldots, \Phi_{K l}\left(w_{*}\left(z_{*}\right)\right)
$$


should be regular in $\mathcal{O}_{o, \mathbb{C}^{M+1}}$, and moreover the quadratic form $q_{2}\left(z_{*}\right)$ should be non-degenerate.

(R2.2) The regularity condition for a singular point on the ramification divisor. Here $w_{0}=0, q_{1}=z_{M+1}, w_{1}=\lambda z_{M+1}$, where $\lambda \in \mathbb{C}$ is a constant, that is, the point $p \in G$ is non-singular. We require the quadratic form $\bar{w}_{2}\left(z_{1}, \ldots, z_{M}\right)$ to have the maximal rank $M$ and the sequence

$$
\bar{q}_{2}, \ldots, \bar{q}_{m}, \bar{w}_{2}, \ldots, \bar{w}_{K}
$$

to be regular in $\mathcal{O}_{o, \mathbb{C}^{M}}$, and moreover, the system of homogeneous equations

$$
\bar{q}_{2}=\cdots=\bar{q}_{m}=\bar{w}_{2}=\cdots=\bar{w}_{K}=0
$$

should define a closed algebraic set in $\mathbb{C}^{M}$, neither component of which is contained in a hyperplane.

Definition 0.3. A Fano cyclic cover $F \in \mathcal{F}$ is said to be regular, if every point $o \in F$ satisfies the corresponding regularity condition $(\mathrm{R} \alpha . \beta)$.

The set of regular cyclic covers will be denoted by the symbol $\mathcal{F}_{\text {reg }}$. Obviously, $\mathcal{F}_{\text {reg }} \subset \mathcal{F}$ is an open set, and a variety $F \in \mathcal{F}_{\text {reg }}$ has at most isolated singularities.

Theorem 2. (i) The set $\mathcal{F}_{\text {reg }}$ is non-empty and moreover, the estimate $\operatorname{codim}_{\mathcal{F}}$ $\left(\mathcal{F} \backslash \mathcal{F}_{\text {reg }}\right) \geq 2$ holds

(ii) If $o \in F$ is a singular point, $F \in \mathcal{F}_{\text {reg }}$, then it is factorial. Setting $H=-K_{F}$, we get $\operatorname{Pic} F=\mathbb{Z} H, A^{2} F=\mathbb{Z} H^{2}$.

Proof of Theorem 2 is given below, in $\S 5$.

Theorem 2 implies the following important conclusion. Let $\pi: V \rightarrow \mathbb{P}^{1}$ be a fibration into Fano cyclic covers, that is, every fiber $F_{t}=\pi^{-1}(t) \in \mathcal{F}$. The fiber space $V / \mathbb{P}^{1}$ can be interpreted as a one-dimensional family of fibers $\left\{F_{t}, t \in \mathbb{P}^{1}\right\}$, that is, a curve in the space $\mathcal{F}$. Now, if the family of fiber spaces $V / \mathbb{P}^{1}$ is sufficiently large (and we consider such families only, see $\S 4$ ), then the corresponding family of curves in $\mathcal{F}$ is sufficiently movable, in particular, a general curve of the family avoids the closed set $\mathcal{F} \backslash \mathcal{F}_{\text {reg. }}$. In other words, for a general fiber space $V / \mathbb{P}^{1}$ in the family under consideration every fiber is regular: $F_{t} \in \mathcal{F}_{\text {reg }}$ for any $t \in \mathbb{P}^{1}$.

Now everything is ready to formulate the main theorem of the present paper. 
0.5. Formulation of the main result. Let $\pi: V \rightarrow \mathbb{P}^{1}$ be a fibration into Fano cyclic covers, $F_{t} \in \mathcal{F}$, where $V$ is a smooth $(M+1)$-dimensional variety, satisfying the conditions

$$
A^{1} V=\operatorname{Pic} V=\mathbb{Z} K_{V} \oplus \mathbb{Z} F, A^{2} V=\mathbb{Z} K_{V}^{2} \oplus \mathbb{Z} H_{F},
$$

where $H_{F}=\left(-K_{V} \cdot F\right)$ is the ample anticanonical section of the fiber, and the fibers $F_{t}, t \in \mathbb{P}^{1}$, have at most isolated factorial singularities. Depending on the context, the hyperplane section of the fiber $H_{F}$ will be considered either as an element of $A^{2} V$, or as an element of $A^{1} F=\operatorname{Pic} F=\mathbb{Z} K_{F}$, where $F=F_{t}$ is a fiber. We get obviously $A^{2} F=\mathbb{Z} H_{F}^{2}$. The symbols $A_{+}^{1} V, A_{+}^{2} V$ and $A_{\text {mov }}^{1} V$ were introduced above (Sec. 0.2). The main result of this paper is the following

Theorem 3. Assume that the Fano fiber space $V / \mathbb{P}^{1}$ satisfies the generalized $K^{2}$-condition of depth 2 , that is,

$$
K_{V}^{2}+2 H_{F} \notin \operatorname{Int} A_{+}^{2} V
$$

and the regularity condition: $F_{t} \in \mathcal{F}_{\text {reg }}$ for any $t \in \mathbb{P}^{1}$. Then for any movable linear system $\Sigma \subset\left|-n K_{V}+l F\right|$ with $l \in \mathbb{Z}_{+}$its virtual and actual thresholds of canonical adjunction coincide: $c_{\mathrm{virt}}(\Sigma)=c(\Sigma)=n$. In particular, if the variety $V$ satisfies the $K$-condition, that is,

$$
-K_{V} \notin \operatorname{Int} A_{\mathrm{mov}}^{1} V
$$

then the variety $V$ is birationally superrigid.

Apart from that, we check birational superrigidity of several families which do not satisfy the $K$-condition, and describe certain explicit constructions for non-rigid cases $(\S 4)$.

The remaining part of the paper contains mainly a proof of Theorem 3. Let us describe briefly its principal steps.

0.6. The structure of the proof. First of all $(\S 1)$, we prove a sufficient condition of birational superrigidity for arbitrary Fano fiber spaces $V / \mathbb{P}^{1}$, that is, we do not assume, that the fibers $F_{t}, t \in \mathbb{P}^{1}$, belong to some particular family of Fano varieties. We assume only that $V$ is a smooth variety, the conditions (4) hold, where $H_{F}=\left(-K_{V} \cdot F\right)$ is the ample anticanonical section of the fiber, and the fibers $F_{t}, t \in \mathbb{P}^{1}$, have at most isolated factorial singularities, and moreover, 
Pic $F_{t}=A^{1} F_{t}=\mathbb{Z} K_{F_{t}}$ and $A^{2} F_{t}=\mathbb{Z} K_{F_{t}}^{2}$ for every $t \in \mathbb{P}^{1}$. The symbols $A_{+}^{1} V$, $A_{+}^{2} V$ and $A_{\text {mov }}^{1} V$ mean the same as above.

So let us formulate the sufficient condition of birational superrigidity. By a degree of an irreducible variety $Y \subset V$, which is contained in a fiber, $Y \subset F_{t}$ (we refer to such varieties as vertical), we mean the number

$$
\operatorname{deg} Y=\left(Y \cdot\left(-K_{V}\right)^{\operatorname{dim} Y}\right)
$$

By a degree of an irreducible subvariety $Y \subset V$, covering the base $\mathbb{P}^{1}, \pi(Y)=\mathbb{P}^{1}$ (we refer to such varieties as horizontal), we mean the number

$$
\operatorname{deg} Y=\left(Y \cdot F \cdot\left(-K_{V}\right)^{\operatorname{dim} Y-1}\right) .
$$

Definition $0.4[\mathbf{2 3}, \mathbf{2 9}, \mathbf{3 0}]$. The fiber space $V / \mathbb{P}^{1}$ satisfies

- the condition $(v)$, if for every irreducible vertical subvariety $Y$ of codimension two (that is, $Y \subset F_{t}$ is a prime divisor, $t=\pi(Y)$ ) and every smooth point $o \in F_{t}$ the inequality

$$
\frac{\operatorname{mult}_{o} Y}{\operatorname{deg} Y} \leq \frac{2}{\operatorname{deg} V}
$$

holds;

- the condition $(f)$, if for every irreducible vertical subvariety $Y$ of codimension three (that is, $\operatorname{codim}_{F} Y=2, F=F_{t} \supset Y$ ) and every smooth point of the fiber $o \in F$, the inequality

$$
\frac{\operatorname{mult}_{o} Y}{\operatorname{deg} Y} \leq \frac{4}{\operatorname{deg} V}
$$

holds.

For the convenience of notations we write down in the sequel the ratio of the multiplicity to the degree by the single symbol

$$
\frac{\operatorname{mult}_{o}}{\operatorname{deg}} Y=\frac{\operatorname{mult}_{o} Y}{\operatorname{deg} Y} .
$$

Let $\Sigma \subset\left|-n K_{V}+l F\right|$ be a movable linear system, $l \in \mathbb{Z}_{+}$. Recall [15,19-24] the following important

Definition 0.5. An exceptional divisor $E$ of a birational morphism $\varphi: \widetilde{V} \rightarrow$ $V$, where $\widetilde{V}$ is a smooth projective variety (we could restrict ourselves by the morphisms $\varphi$ of the form $\varphi_{N} \circ \cdots \circ \varphi_{1}$, where $\varphi_{i}$ are blow ups with non-singular 
centres), is a maximal singularity of the linear system $\Sigma$, if the Noether-Fano inequality holds:

$$
\nu_{E}(\Sigma)>n a(E)
$$

where $\nu_{E}(\Sigma)=\operatorname{ord}_{E} \varphi^{*} \Sigma$ is the multiplicity of the full preimage of a general divisor of the system $\Sigma$ along $E, a(E)$ is the discrepancy, $n \in \mathbb{Z}_{+}$has been defined above. For $n \geq 1$ the inequality (7) means that the pair $\left(V, \frac{1}{n} \Sigma\right)$ is not canonical and $E \subset \widetilde{V}$ is its non-canonical singularity. The irreducible subvariety

$$
B=\varphi(E)=\text { centre }(E, V)
$$

is called the centre of the non-canonical (maximal) singularity $E$.

Theorem 4. Assume that the Fano fiber space $V / \mathbb{P}^{1}$ satisfies the generalized $K^{2}$-condition of depth 2, that is,

$$
K_{V}^{2}+2 H_{F} \notin \operatorname{Int} A_{+}^{2} V,
$$

and the conditions $(v)$ and $(f)$, formulated above.

(i) If the centre of every maximal singularity of a movable linear system $\Sigma \subset$ $\left|-n K_{V}+l F\right|$ with $l \in \mathbb{Z}_{+}$is not a singularity of a fiber, then the virtual and actual thresholds of canonical adjunction of the system $\Sigma$ coincide: $c_{\mathrm{virt}}(\Sigma)=c(\Sigma)$.

(ii) If the assumption (i) holds for any movable linear system on $V$ and the variety $V$ satisfies the $K$-condition, that is,

$$
-K_{V} \notin \operatorname{Int} A_{\mathrm{mov}}^{1} V
$$

then the variety $V$ is birationally superrigid.

Proof of the theorem is given below in $\S 1$. Now we just note that the claim (ii) follows from (i) in an obvious way. The theorem reduces proving birational superrigidity to checking the $K^{2}$-condition, the $K$-condition, the conditions $(v)$ and $(f)$ and, finally, excluding the maximal singularities, the centre of which is a singular point of a fiber. Here

- the $K^{2}$ - and $K$-conditions are checked in a routine way, usually this is not hard to do, and, in a sense, the "majority" of Fano fiber spaces satisfy these conditions;

- as a rule, the conditions $(v)$ and $(f)$ are known, if every smooth fiber is birationally superrigid, since it is by checking the inequality (6) in the condition $(f)$ that one proves superrigidity of a Fano variety, whereas the 
condition $(v)$, that is, the inequality (5), usually follows from $(f)$ in a straightforward way (see below);

- it is the exclusion of a maximal singularity, lying over a singular point of a fiber, that always made the hardest problem and for this reason was the key part of the proof [23,29], however employing the connectedness principle of Shokurov and Kollár [16,43], it is possible to essentially simplify this check, similar to the way in which it is done below, even relaxing the conditions of general position compared to $[23,29]$.

Having proved in $\S 1$ the criterion of birational superrigidity, to show Theorem 3 it is sufficient to check that the fiber spaces $V / \mathbb{P}^{1}$ satisfy all the conditions of Theorem 4. In order to do that, we develop in $\S 2$, following $[21,25,28]$, but in a somewhat more general form, the technique of hypertangent divisors. The first applications of this technique are the proof of Theorem 1 (birational superrigidity in the absolute case) and exclusion of maximal singularities lying over a double point of a fiber outside the ramification divisor.

To complete the proof of Theorem 3, it remains to exclude the maximal singularities, lying over a double point of a fiber on the ramification divisor. It is done in $\S 3$ using the connectedness principle of Shokurov and Kollár and the technique of hypertangent divisors. Note that this fragment of the proof can be realized without using the connectedness principle, similar to the arguments in [29, §3]. However, the connectedness principle makes it possible not only to simplify the proof and make it shorter, but also to relax the regularity conditions imposed on the singularity of the fiber. This method of exclusion of maximal singularities was earlier used in $[33, \S 3]$.

This completes the proof of the main Theorem 3.

The remaining two sections are devoted, respectively, to an explicit construction of the fiber spaces $V / \mathbb{P}^{1}$ and to checking the $K^{2}$-condition and the $K$-condition $(\S 4)$ and to proving the regularity conditions, that is, Theorem $2(\S 5)$.

Remark 0.2. (i) The arguments of this paper remain true (with considerable simplifications), if $m=1$, that is, the fiber of the fiber space $V / \mathbb{P}^{1}$ is a Fano cyclic space of index 1 . We excluded this case from consideration because for this case it would be natural to somewhat change the notations: to forget about the hypersurface $Q \subset X$ and construct $V$ as a cyclic cover $\sigma: V \rightarrow X$, branched 
over a smooth divisor $W \subset X$. However, if the notations were changed, we would have to consider the case of a pencil of cyclic spaces separately from the general case, which would have made the structure of the paper unnecessary complicated. We leave the formulation and proof of Theorem 3, and also modification of the explicit constructions of $\S 4$ for the case of cyclic spaces to the reader.

(ii) In this paper all the necessary work is done that makes it possible to describe the structures of fibrations into varieties of zero Kodaira dimension on $V$. For the modifications needed, see $[34,42]$.

0.7. Historical remarks. Development of the method of maximal singularities in all details was traced down in the introductions to the papers [21,28-30]. Here we just remind the main milestones of this progress. On the modern stage of algebraic geometry the starting point of the method of maximal singularities was the pioneer paper of V.A.Iskovskikh and Yu.I.Manin on the three-dimensional quartic [15]. V.G.Sarkisov obtained the first result in dimension three and higher in the relative case, for the conic bundles [35,36]. A systematic work with Fano varieties of arbitrary dimension became possible only after the technique of hypertangent divisors was introduced [21], and with Fano fiber spaces over $\mathbb{P}^{1}$ only after the paper [20]. In the last decade the progress in this field considerably accelerated, see [3,6-8,10-13,20-34,37,38,40-42]. Attempts to employ the technique of the minimal model program resulted in some enlarging of the class of varieties under consideration [6-8] and in a greater diversity of ideas in the field $[3,6,27,40,41]$, however it was only in $[32,33]$ that the use of the connectedness principle of Shokurov and Kollár made it possible to obtain essentially new results that were out of reach for the old technique, and simplify and clarify certain earlier arguments.

In this paper the method of maximal singularities is presented in its most upto-day version, including both the traditional quadratic technique of counting multiplicities and the technique of hypertangent divisors and the linear method of excluding maximal singularities based on the connectedness principle.

This version is certainly not a final one, and hopefully in the future will be improved further on. 


\section{The method of maXimal Singularities}

In this section we prove Theorem 4. Essentially, the method of maximal singularities reduces the problem of birational rigidity of a Fano fiber space $V / \mathbb{P}^{1}$ to certain problems of numerical geometry of its fibers and numerical characteristics of "twistedness" of the fiber space $V / \mathbb{P}^{1}$ over the base. In this section we prove Theorem 4, which gives a sufficient condition of birational superrigidity and presents a version of such reduction. Another versions of the method see in [23,29].

Recall that in this section we consider Fano fiber spaces $V / \mathbb{P}^{1}$ not assuming, that the fibers $F_{t}, t \in \mathbb{P}^{1}$, belong to some particular family of Fano varieties. We assume only that $V$ is a smooth variety, the conditions (4) hold, where $H_{F}=$ $\left(-K_{V} \cdot F\right)$ is the ample anticanonical section of the fiber, and the fibers $F_{t}, t \in \mathbb{P}^{1}$, have at most isolated factorial singularities, and moreover, that Pic $F_{t}=A^{1} F_{t}=$ $\mathbb{Z} K_{F_{t}}$ and $A^{2} F_{t}=\mathbb{Z} K_{F_{t}}^{2}$ for any $t \in \mathbb{P}^{1}$. The symbols $A_{+}^{1} V, A_{+}^{2} V$ and $A_{\text {mov }}^{1} V$ were introduced above.

1.1. Start of the proof of Theorem 4. Fix a movable linear system $\Sigma \subset$ $\left|-n K_{V}+l F\right|$ with $l \in \mathbb{Z}_{+}$. Obviously, $c(\Sigma)=n$, where $n=0$ if and only if $\Sigma$ is composed from the pencil of fibers $|F|$. Assume that the inequality

$$
c_{\text {virt }}(\Sigma)<n
$$

holds, in particular, $n \geq 1$. This means that there exists a birational morphism $\varphi: \widetilde{V} \rightarrow V$ of smooth varieties such that

$$
c(\widetilde{\Sigma}, \widetilde{V})<n
$$

where $\widetilde{\Sigma}$ is the strict transform of $\Sigma$ on $\widetilde{V}$. This implies immediately that the pair $\left(V, \frac{1}{n} \Sigma\right)$ is not canonical (see [20,23]), that is, the system $\Sigma$ has a maximal singularity $E \subset \widetilde{V}$. Consider its centre $B=\varphi(E) \subset V$, an irreducible subvariety of codimension at least two.

Let

$$
\begin{aligned}
\varphi_{i, i-1}: & V_{i} \rightarrow V_{i-1} \\
& \bigcup \bigcup \\
& E_{i} \rightarrow B_{i-1}
\end{aligned}
$$

be a sequence of blow ups with irreducible centres $B_{i-1} \subset V_{i-1}$, which is uniquely determined by the following conditions: 
1) $V_{0}=V, B_{0}=B, i=1, \ldots, N$

2) $B_{j}=\operatorname{centre}\left(E, V_{j}\right) \subset V_{j}, E_{j+1}=\varphi_{j+1, j}^{-1}\left(B_{j}\right)$;

3) the valuation $\nu_{E_{N}}$ coincides with $\nu_{E}$.

In other words, the birational map $V_{N} \rightarrow \widetilde{V}$ is biregular at the generic point of the divisor $E_{N}$ and transforms $E_{N}$ into $E$. Note that the varieties $V_{1}, \ldots, V_{N}$, generally speaking, can be singular, since the centres $B_{j}$ of blow ups are not necessarily smooth. However, $V_{j}$ is smooth at the generic point of $B_{j}$. By the symbol $\Sigma^{j}$ we denote the strict transform of the linear system $\Sigma$ on $V_{j}$. Set

$$
\nu_{j}=\operatorname{mult}_{B_{j-1}} \Sigma^{j-1}, \quad \delta_{j}=\operatorname{codim} B_{j-1}-1 .
$$

On the set of exceptional divisors

$$
\left\{E_{1}, \ldots, E_{N}\right\}
$$

we define in the usual way $[15,19-24,30]$ the structure of an oriented graph: $E_{i}$ and $E_{j}$ are joined by an oriented edge (an arrow), if and only if $i>j$ and

$$
B_{i-1} \subset E_{j}^{i-1}
$$

notation: $i \rightarrow j$. As usual, set for $i>j$

$$
p_{i j}=\sharp\left\{\text { the paths from } E_{i} \text { to } E_{j}\right\} \geq 1,
$$

$p_{i i}=1$ by definition. Set $p_{i}=p_{N i}$. The Noether-Fano inequality takes the traditional form:

$$
\sum_{i=1}^{N} p_{i} \nu_{i}>n \sum_{i=1}^{N} p_{i} \delta_{i} .
$$

Proposition 1.1. The centre $B$ of the maximal singularity $E$ on $V$ is contained in some fiber $F_{t}=\pi^{-1}(t), t \in \mathbb{P}^{1}$.

Proof. Assume the converse: $\pi(B)=\mathbb{P}^{1}$. Let $F \subset V$ be a fiber of general position. It is easy to see that the restriction $\Sigma_{F}=\left.\Sigma\right|_{F}$ of the linear system $\Sigma$ onto $F$ is a movable linear system $\Sigma_{F} \subset\left|n H_{F}\right|$, and moreover the pair $\left(F, \frac{1}{n} \Sigma_{F}\right)$ is not canonical, that is, $\Sigma_{F}$ has a maximal singularity. The easiest way to define this maximal singularity is as follows: let $\widetilde{F} \subset \widetilde{V}$ be the strict transform, $E_{F}$ an irreducible component of the closed set $\widetilde{F} \cap E$. Otherwise, one can restrict the sequence of blow ups (8) onto the fiber $F$. The discrepancy remains the same: $a\left(E_{F}, F\right)=a(E, V)$. The centre of the singularity $E_{F}$ is $\varphi\left(E_{F}\right)$, an irreducible component of the closed set $B \cap F$. 
By assumption, Pic $F=\mathbb{Z} K_{F}, A^{2} F=\mathbb{Z} K_{F}^{2}$, and for every irreducible subvariety $Y \subset F$ of codimension two at every point the inequality (6) holds. The standard fact of the theory of birational rigidity: these two assumptions imply the every pair $\left(F, \frac{1}{n} \Sigma_{F}\right)$ is canonical, where $\Sigma_{F} \subset\left|n H_{F}\right|$ is a movable linear system. This contradiction completes the proof of the proposition.

Thus $B \subset F=F_{t}$ is contained in a fiber.

Lemma 1.1. The following inequality holds: $\operatorname{codim}_{F} B \geq 2$.

Proof. Assume the converse: $B \subset F$ is a prime divisor. Let $D \in \Sigma$ be a general divisor, $D_{F}$ its restriction onto $F$. By the Noether-Fano inequality $\operatorname{mult}_{B} D>n$, so that $D_{F}=\alpha B+D^{\sharp}$, where $\alpha>n$ and $D^{\sharp}$ is an effective divisor on $F$. However, $D_{F} \sim n H_{F}$, which immediately gives a contradiction. Q.E.D. for the lemma.

Let $\mathcal{M}=\left\{T_{1}, \ldots, T_{k}\right\}$ be the set of all maximal singularities of the linear system $\Sigma$ on $\widetilde{V}$. As we have proved above, the centre $B_{E}=\operatorname{centre}\left(\nu_{E}\right)$ of any maximal singularity $E \in \mathcal{M}$ is contained in some fiber $F_{t}$. The set $\mathcal{M}$ is finite, so that there are at most finitely many points $t \in \mathbb{P}^{1}$, the fibers $F_{t}$ over which contain a centre of a maximal singularity. Set $\mathcal{M}_{t}=\left\{E \in \mathcal{M} \mid B_{E} \subset F_{t}\right\}$,

$$
e(E)=\nu_{E}(\Sigma)-n a(E, V)>0
$$

for $E \in \mathcal{M}$. Recall that $\Sigma \subset\left|-n K_{V}+l F\right|, l \in \mathbb{Z}_{+}$.

Proposition 1.2. The following inequality holds:

$$
\sum_{t \in \mathbb{P}^{1}} \max _{\left\{E \in \mathcal{M}_{t}\right\}} \frac{e(E)}{\nu_{E}\left(F_{t}\right)}>l
$$

Proof. Let $\widetilde{D} \in \widetilde{\Sigma}$ be a general divisor, that is, the strict transform of a divisor $D \in \Sigma$ of general position on $\widetilde{V}$. By assumption, the linear system $|\widetilde{D}+n \widetilde{K}|$ is empty (where $\widetilde{K}$ is the canonical class of the variety $\widetilde{V}$ ). Therefore the linear system

$$
\left|l F-\sum_{E \in \mathcal{M}} e(E) E\right|
$$

is empty, either. On the other hand, for $E \in \mathcal{M}_{t}$ by construction the divisor

$$
F_{t}-\nu_{E}\left(F_{t}\right) E
$$


is effective, so that the divisor

$$
\sum_{t \in \mathbb{P}_{1}}\left[\left(\max _{\left\{E \in \mathcal{M}_{t}\right\}} \frac{e(E)}{\nu_{E}\left(F_{T}\right)}\right) F_{t}-\sum_{E \in \mathcal{M}_{t}} e(E) E\right]
$$

is also effective. From this, we immediately obtain the inequality (9).

Q.E.D. for Proposition 1.2.

1.2. The structure of the sequence of blow ups. Consider the selfintersection of the linear system $\Sigma$, that is, the effective algebraic cycle $Z=$ $\left(D_{1} \circ D_{2}\right)$, where $D_{1}, D_{2} \in \Sigma$ are general divisors. Let $Z=Z^{v}+Z^{h}$ be the decomposition of the cycle $Z$ into the vertical $\left(Z^{v}\right)$ and horizontal $\left(Z^{h}\right)$ parts. The cycle $Z^{v}$ can be further decomposed as

$$
Z^{v}=\sum_{t \in \mathbb{P}^{1}} Z_{t}^{v}, \quad \operatorname{Supp} Z_{t}^{v} \subset F_{t} .
$$

Let $E \in \mathcal{M}_{t}$ be a maximal singularity over a point $t \in \mathbb{P}^{1}$. To prove Theorem 4 , we apply to the effective cycle $Z_{t}^{v}+Z^{h}$ the technique of counting multiplicities in the form of the paper [30]. First of all, let us study the structure of the sequence of blow ups, resolving the singularity $E$. This means, to break the sequence of blow ups into segments that determine multiplicities of the cycles $Z_{t}^{v}, Z^{h}$ and $\left(Z^{h} \circ F_{t}\right)$ (the intersection of a horizontal cycle with a fiber is always well defined).

So long as we discuss a fixed singularity $E$, we omit the symbols $t$ and $E$ for simplicity of notations, for instance, we write $F$ instead of $F_{t}, Z^{v}$ instead of $Z_{t}^{v}$, $e$ instead of $e(E)$ and so on.

So let us consider the sequence of blow ups (8), associated with E. As usual, the upper index $j$ means the operation of taking the strict transform on $V_{j}$, for instance, $F^{j} \subset V_{j}$ is the strict transform of the fiber $F$ and so on. Set

$$
N_{f}=\max \left\{i \mid B_{i-1} \subset F^{i-1}\right\} .
$$

Since $\varphi_{i, i-1}\left(B_{i}\right)=B_{i-1}$ for any $i=1, \ldots N-1$, the codimensions $\operatorname{codim} B_{i}$ do not increase. Set

$$
L=\max \left\{i \mid \operatorname{codim} B_{i-1} \geq 3\right\} \leq N .
$$

We introduce the following notations: for $i \in\{1, \ldots, L\}$

$$
m_{i}^{h}=\operatorname{mult}_{B_{i-1}}\left(Z^{h}\right)^{i-1}, \quad m_{i}^{v}=\operatorname{mult}_{B_{i-1}}\left(Z^{v}\right)^{i-1},
$$


$m_{i}^{h(v)} \leq m_{i-1}^{h(v)}$ for $i=2, \ldots, L$. Note that by the assumption (i) of Theorem 4 the fiber $F$ can be assumed to be smooth at the generic point of $B$ and therefore the strict transform $F^{i}$ is smooth at the generic point of each variety $B_{i}$, if $B_{i} \subset F^{i}$. Thus for $i \in\left\{1, \ldots, N_{f}\right\}$ we get $\operatorname{mult}_{B_{i-1}} F^{i-1}=1$. For $i>N_{f}$, obviously, mult $_{B_{i-1}} F^{i-1}=0$. The more so, $m_{i}^{v}=0$ for $i>N_{f}$ (if $N_{f}<L$ ). Now the technique of counting multiplicities $[19,21,24]$ combined with the relation

$$
\sum_{i=1}^{K} p_{i} \nu_{i}=n \sum_{i=1}^{K} p_{i} \delta_{i}+e
$$

$e>0$, gives the inequality

$$
\sum_{i=1}^{L} p_{i} m_{i}^{h}+\sum_{i=1}^{\min \left\{N_{f}, L\right\}} p_{i} m_{i}^{v} \geq \sum_{i=1}^{N} p_{i} \nu_{i}^{2} \geq \frac{\left(n \sum_{i=1}^{N} p_{i} \delta_{i}+e\right)^{2}}{\sum_{i=1}^{N} p_{i}}
$$

where $p_{i}$ is the number of paths in the graph $\Gamma$ of the resolution of the maximal singularity $E$ going from the vertex $E_{N}$ to $E_{i}$.

Unfortunately, the estimate (10) is not strong enough for our purposes (it would have been sufficient under the assumption that the standard $K^{2}$-condition holds, $K_{V}^{2} \notin \operatorname{Int} A_{+}^{2} V$, but in Theorem 4 a weaker assumption, the $K^{2}$-condition of depth 2 , is used). A more refined study of the resolution of the singularity $E$ is needed. Set

$$
\Sigma_{l}=\sum_{i=1}^{L} p_{i}, \quad \Sigma_{u}=\sum_{i=L+1}^{N} p_{i}, \quad \Sigma_{f}=\sum_{i=1}^{\min \left\{N_{f}, L\right\}} p_{i} .
$$

Note that $\nu_{E}(F)=\sum_{i=1}^{N_{f}} p_{i} \geq \Sigma_{f}$. Obviously,

$$
m_{i}^{h} \leq m_{h}=m_{1}^{h}=\operatorname{mult}_{B} Z^{h}
$$

Set also

$$
d_{h}=\operatorname{deg} Z^{h}, \quad d_{v}=\operatorname{deg} Z_{t}^{v} .
$$

Now let us break the set of blow ups into a few subsets. First of all, let us separate the blow ups of subvarieties $B_{i-1}$ of codimension three. Set

$$
\begin{gathered}
J_{s}=\left\{i \mid 1 \leq i \leq K, \quad \operatorname{codim} B_{i-1} \geq 4\right\}, \\
J_{m}=\left\{i \mid 1 \leq i \leq K, \quad \operatorname{codim} B_{i-1}=3\right\}
\end{gathered}
$$




$$
J_{u}=\{i \mid L+1 \leq i \leq K\}, \quad J_{l}=J_{s} \cup J_{m} .
$$

In its turn, we break the set $J_{m}$ into two disjoint subsets, $J_{m}=J_{m}^{+} \amalg J_{m}^{-}$, where

$$
J_{m}^{+}=\left\{i \in J_{m} \mid B_{i-1} \subset F^{i-1}\right\},
$$

$J_{m}^{-}=J_{m} \backslash J_{m}^{+}=\left\{i \in J_{m} \mid B_{i-1} \not \subset F^{i-1}\right\}$. It can well turn out that $J_{m}^{+}$or $J_{m}^{-}$(or the whole set $J_{m}$ ) is empty. Set, furthermore,

$$
\Sigma_{s}=\sum_{i \in J_{s}} p_{i}, \quad \Sigma_{m}^{ \pm}=\sum_{i \in J_{m}^{ \pm}} p_{i}, \quad \Sigma_{m}=\Sigma_{m}^{+}+\Sigma_{m}^{-},
$$

the symbol $\Sigma_{u}$ retains its meaning. Obviously, $\Sigma_{l}=\Sigma_{s}+\Sigma_{m}$.

Now the inequality (10) can be rewritten as

$$
\sum_{i \in J_{l}} p_{i} m_{i}^{h}+\sum_{i \in J_{s} \cup J_{m}^{+}} p_{i} m_{i}^{v} \geq \frac{\left(\left(3 \Sigma_{s}+2 \Sigma_{m}+\Sigma_{u}\right) n+e\right)^{2}}{\Sigma_{s}+\Sigma_{m}+\Sigma_{u}} .
$$

The next step is estimating the horizontal multiplicities $m_{i}^{h}$.

Proposition 1.3. The following inequality holds:

$$
\sum_{i \in J_{s} \cup J_{m}^{+}} p_{i} m_{i}^{h} \leq \Sigma_{s} \operatorname{mult}_{B}\left(Z^{h} \circ F\right) .
$$

1.3. Multiplicities of the horizontal cycles. Let us prove Proposition 1.3. The arguments below hold with obvious simplifications when $J_{m}^{+}=\emptyset$. So we assume that $J_{m}^{+} \neq \emptyset$, so that, in particular, $J_{s} \subset\left\{1, \ldots, N_{f}\right\}$.

First let us consider the following general situation. Let $Y \subset V$ be an irreducible horizontal subvariety of codimension two, $Y^{i} \subset V_{i}$ its strict transform,

$$
m_{Y}(i)=\operatorname{mult}_{B_{i-1}} Y^{i-1}
$$

the corresponding multiplicity. Set $Y_{F}=(Y \circ F)$. It is an effective cycle of codimension two in the fiber $F$. Let $Y_{F}^{i} \subset V_{i}$ be its strict transform,

$$
m_{Y, F}(i)=\operatorname{mult}_{B_{i-1}} Y_{F}^{i-1} .
$$

Since the support of the cycle $Y_{F}$ is contained in the fiber $F$, the numbers $m_{Y, F}(i)$ vanish for $i \in J_{m}^{-}$. 
Lemma 1.2. The following estimate holds:

$$
\sum_{i \in J_{s} \cup J_{m}^{+}} p_{i} m_{Y}(i) \leq \sum_{i \in J_{s}} p_{i} m_{Y, F}(i) .
$$

Before starting the proof, recall some facts which follow immediately from the elementary intersection theory [39]. Note that here we intersect a divisor and a subvariety of arbitrary codimension, unlike [19,24], where the case of two divisors was considered. Let $X$ be an arbitrary smooth variety, $B \subset X, B \not \subset \operatorname{Sing} X$ an irreducible subvariety of codimension $\geq 2, \sigma_{B}: X(B) \rightarrow X$ its blow up, $E(B)=$ $\sigma_{B}^{-1}(B)$ the exceptional divisor. Let

$$
Z=\sum m_{i} Z_{i}, \quad Z_{i} \subset E(B),
$$

be a cycle of dimension $k, k \geq \operatorname{dim} B$. Define the degree of the cycle $Z$, setting

$$
\operatorname{deg} Z=\sum_{i} m_{i} \operatorname{deg}\left(Z_{i} \bigcap \sigma_{B}^{-1}(b)\right),
$$

where $b \in B$ is a point of general position, $\sigma_{B}^{-1}(b) \cong \mathbb{P}^{\text {codim } B-1}$ and the degree in the right-hand side is the usual degree in the projective space.

Note that $\operatorname{deg} Z_{i}=0$ if and only if $\sigma_{B}\left(Z_{i}\right)$ is a proper closed subset of the subvariety $B$.

Now let $D$ be a prime Weil divisor on $X, Y \subset X$ an irreducible subvariety of $\operatorname{dimension} l \leq \operatorname{dim} X-1$. Assume that $Y \not \subset D$ and that $\operatorname{dim} B \leq l-1$. The strict transforms of the divisor $D$ and the subvariety $Y$ on $X(B)$ denote by the symbols $D^{B}$ and $Y^{B}$, respectively.

Lemma 1.3. (i) Assume that $\operatorname{dim} B \leq l-2$. Then

$$
D^{B} \circ Y^{B}=(D \circ Y)^{B}+Z,
$$

where o means the operation of taking the algebraic cycle of the scheme-theoretic intersection, Supp $Z \subset E(B)$ and

$$
\operatorname{mult}_{B}(D \circ Y)=\operatorname{mult}_{B} D \cdot \operatorname{mult}_{B} Y+\operatorname{deg} Z .
$$

(ii) Assume that $\operatorname{dim} B=l-1$. Then

$$
D^{B} \circ Y^{B}=Z+Z_{1},
$$

where $\operatorname{Supp} Z \subset E(B)$, Supp $\sigma_{B}\left(Z_{1}\right)$ does not contain $B$ and

$$
D \circ Y=\left[\left(\operatorname{mult}_{B} D\right)\left(\operatorname{mult}_{B} Y\right)+\operatorname{deg} Z\right] B+\left(\sigma_{B}\right)_{*} Z_{1} .
$$


Proof is easy to obtain by the standard intersection theory [39].

1.4. The technique of counting multiplicities. Let us construct a sequence of effective cycles of codimension three on the varieties $V_{i}$, setting

$$
\begin{aligned}
Y & \circ F=Z_{0}\left(=Y_{F}\right), \\
Y^{1} & \circ F^{1}=Z_{0}^{1}+Z_{1}, \\
\quad & \\
Y^{i} & \circ F^{i}=\left(Y^{i-1} \circ F^{i-1}\right)^{i}+Z_{i},
\end{aligned}
$$

$i \in J_{s}$, where $\operatorname{Supp} Z_{i} \subset E_{i}$. Thus for every $i \in J_{s}$ we get:

$$
Y^{i} \circ F^{i}=Y_{F}^{i}+Z_{1}^{i}+\cdots+Z_{i-1}^{i}+Z_{i} .
$$

For any $j>i, j \in J_{s}$ set

$$
m_{i, j}=\operatorname{mult}_{B_{j-1}}\left(Z_{i}^{j-1}\right)
$$

(the multiplicity of an irreducible subvariety along a smaller subvariety is understood in the usual sense; for an arbitrary cycle we extend the multiplicity by linearity).

Now set $d_{i}=\operatorname{deg} Z_{i}$. We obtain the following system of equalities:

$$
\begin{aligned}
& m_{Y}(1)+d_{1}=m_{Y, F}(1), \\
& m_{Y}(2)+d_{2}=m_{Y, F}(2)+m_{1,2}, \\
& \quad \vdots \\
& m_{Y}(i)+d_{i}=m_{Y, F}(i)+m_{1, i}+\cdots+m_{i-1, i}
\end{aligned}
$$

$i \in J_{s}$. Setting $S=\max \left\{i \in J_{s}\right\}$, consider the last equality in this sequence:

$$
m_{Y}(S)+d_{S}=m_{Y, F}(S)+m_{1, S}+\cdots+m_{S-1, S} .
$$

If $J_{m}^{+} \neq \emptyset$, then by part (ii) of Lemma 1.3 we get

$$
d_{S} \geq \sum_{i \in J_{m}^{+}} m_{Y}(i) \operatorname{deg}\left(\varphi_{i-1, S}\right)_{*} B_{i-1} \geq \sum_{i \in J_{m}^{+}} m_{Y}(i) .
$$

Now recall the following useful 
Definition 1.1. (see $[19,24])$. A function $a: J_{s} \rightarrow \mathbb{R}_{+}$is said to be compatible with the graph structure, if

$$
a(i) \geq \sum_{\substack{j \rightarrow i, j \in J_{s}}} a(j)
$$

for any $i \in J_{s}$.

In fact, we will use only one function compatible with the graph structure, namely $a(i)=p_{i}$.

Proposition 1.4. Let $a(\cdot)$ be a function, compatible with the graph structure. Then the following inequality holds:

$$
\sum_{i \in J_{s}} a(i) m_{Y, F}(i) \geq \sum_{i \in J_{s}} a(i) m_{Y}(i)+a(S) \sum_{i \in J_{m}^{+}} m_{Y}(i) .
$$

Proof is given word for word in the same way as for the case of two divisors ([19,21,24]): multiply the $i$-th equality by $a(i)$ and put them all together. In the right-hand side for any $i \geq 1$ we get the expression

$$
\sum_{j \geq i+1} a(j) m_{i, j}
$$

In the left-hand side for any $i \geq 1$ we get the summand $a(i) d_{i}$.

Lemma 1.4. If $m_{i, j}>0$, then $j \rightarrow i$.

Proof [19,24]: if $m_{i, j}>0$, then $B_{j-1} \subset \operatorname{Supp} Z_{i}^{j-1}$, but Supp $Z_{i} \subset E_{i}$, so that $B_{j-1} \subset E_{i}^{j-1}$. Q.E.D. for the lemma.

The next standard step is to compare the multiplicities $m_{i, j}$ with the degrees.

Lemma 1.5. For any $i<j \in J_{s}$ we get

$$
m_{i, j} \leq d_{i}
$$

Proof. If $m_{i, j}=0$, then there is nothing to prove. Otherwise, $j \rightarrow i$ and we have to show that

$$
\operatorname{mult}_{B_{j-1}} Z_{i}^{j-1} \leq \operatorname{deg} Z_{i}
$$

Taking into account that the maps $\varphi_{a, b}: B_{a} \rightarrow B_{b}$ are surjective, it is sufficient to prove the inequality

$$
\operatorname{mult}_{\left[B_{j-1} \cap \varphi_{i, i-1}^{-1}(t)^{j-1}\right]}\left[Z_{i} \cap \varphi_{i, i-1}^{-1}(t)\right]^{j-1} \leq \operatorname{deg}\left[Z_{i} \cap \varphi_{i, i-1}^{-1}(t)\right],
$$


where $t \in B_{i-1}$ is a point of general position. Taking into account that $\varphi_{i, i-1}^{-1}(t)$ is the projective space $\mathbb{P}^{\text {codim } B_{i-1}-1}$, we get that in the right-hand side in (17) we get the usual degree of a hypersurface in the projective space, whereas the set $\left[Z_{i} \cap \varphi_{i, i-1}^{-1}(t)\right]^{j-1}$ is obtained from this hypersurface by a finite sequence of blow ups $\varphi_{s, s-1}, s=i+1, \ldots, j-1$, restricted onto $\varphi_{i, i-1}^{-1}(t)$. Taking into account that the multiplicities do not increase under the blow ups, we reduce the claim to the obvious case of a hypersurface in the projective space. Q.E.D. for the lemma.

As a result, we get the following estimate:

$$
\sum_{j \geq i+1} a(j) m_{i, j}=\sum_{\substack{j \geq i+1 \\ m_{i, j} \neq 0}} a(j) m_{i, j} \leq d_{i} \sum_{j \rightarrow i} a(j) \leq a(i) d_{i} .
$$

By what was said above, we can delete in the right-hand side all the summands $m_{i, *}, i \geq 1$, and in the left-hand side all the summands $d_{i}, i \geq 1$, replacing the equality sign $=$ by the inequality $\operatorname{sign} \leq$. Q.E.D.

Setting in the inequality (16) $a(i)=p_{i}$ and taking into account that for $j \geq S$ we have $p_{j} \leq p_{S}$, we complete the proof of Lemma 1.2.

Now let us complete the proof of Proposition 1.3.

Obviously, the inequality (15) remains true, if $Y$ is an effective horizontal cycle of codimension two on $V$, that is, each irreducible component of the cycle $Y$ is a horizontal subvariety. The formulae $(13,14)$ extend by linearity to the set of all effective horizontal cycles, whereas the left-hand side and right-hand side of the inequality (15) are linear in $m_{Y}(\cdot), m_{Y, F}(\cdot)$, respectively.

Now set $Y=Z^{h}$ and take into account that

$$
m_{Y, F}(i) \leq \operatorname{mult}_{B}\left(Z^{h} \circ F\right)
$$

for $i \geq 1$. This proves Proposition 1.3.

1.5. The supermaximal singularity. We apply the estimates obtained above to a maximal singularity $E \in \mathcal{M}$, satisfying, apart from the Noether-Fano inequality, a certain additional condition formulated below in Proposition 1.5. Such singularities are said to be supermaximal. Since by assumption the $K^{2}$-condition of depth 2 holds, for the horizontal part of the self-intersection of the linear system 
$\Sigma$ we get

$$
Z^{h} \sim n^{2} K_{V}^{2}+\alpha H_{F},
$$

where the coefficient $\alpha \in \mathbb{Z}$ satisfies the inequality

$$
\alpha \geq-2 n^{2} .
$$

Therefore, for the vertical component we get

$$
Z^{v} \sim(2 n l-\alpha) H_{F},
$$

whence

$$
\operatorname{deg} Z^{v}=\sum_{t \in \mathbb{P}^{1}} \operatorname{deg} Z_{t}^{v} \leq\left(2 n l+2 n^{2}\right) \operatorname{deg} V
$$

Proposition 1.5. For some point $t \in \mathbb{P}^{1}$ there is a maximal singularity $E \in$ $\mathcal{M}_{t} \neq \emptyset$, satisfying the estimate

$$
e(E)>\frac{\nu_{E}\left(F_{t}\right)}{2}\left(\frac{\operatorname{deg} Z_{t}^{v}}{n \operatorname{deg} V}-2 n\right)
$$

Proof of Proposition 1.5. Compare the inequalities (9) and (18). Replacing the number $l$ in the right-hand side of the inequality (18) by the left-hand side of the inequality (9), we get

$$
\sum_{t \in \mathbb{P}^{1}}\left[\operatorname{deg} Z_{t}^{v}-2 n \operatorname{deg} V \max _{\left\{E \in \mathcal{M}_{t}\right\}} \frac{e(E)}{\nu_{E}\left(F_{t}\right)}\right]<2 n^{2} \operatorname{deg} V,
$$

whence our proposition follows immediately.

Remark 1.1. If there are several maximal singularities, the centres of which lie in the fibers over distinct points $t_{1}, \ldots, t_{k}$, then Proposition 1.5 gets stronger: there is a maximal singularity $E \in \mathcal{M}_{t}, t \in\left\{t_{1}, \ldots, t_{k}\right\}$, satisfying the estimate

$$
e(E)>\frac{\nu_{E}\left(F_{t}\right)}{2}\left(\frac{\operatorname{deg} Z_{t}^{v}}{n \operatorname{deg} V}-\frac{2 n}{k}\right) .
$$

Thus we consider the worst possible case, setting $k=1$.

Let $o \in B$ be a point of general position. Since by assumption $o \in F$ is a smooth point of the fiber, the conditions $(f)$ and $(v)$ hold. From the inequality (6) we immediately get the estimate

$$
\sum_{i \in J_{s} \cup J_{m}^{+}} p_{i} m_{i}^{h} \leq 4 n^{2} \Sigma_{s}
$$


Since $m_{i}^{h} \leq m_{1}^{h} \leq 4 n^{2}$, we get the inequality

$$
\sum_{i \in J_{l}} p_{i} m_{i}^{h} \leq 4 n^{2}\left(\Sigma_{s}+\Sigma_{m}^{-}\right)
$$

This is the very estimate for the singularities of the horizontal component $Z^{h}$ that we need.

Now consider the vertical component $Z^{v}$. By the condition $(v)$ the inequality

$$
m_{i}^{v} \leq m_{1}^{v} \leq \frac{2}{\operatorname{deg} V} d_{v}
$$

holds. On the other hand, the generalized $K^{2}$-condition of depth 2 implies the estimate

$$
\frac{d_{v}}{\operatorname{deg} V}<\frac{2 e n}{\nu_{E}(F)}+2 n^{2}
$$

Combining (21) and (22), we obtain the inequality

$$
\sum_{i \in J_{s} \cup J_{m}^{+}} p_{i} m_{i}^{v}<2 n\left(\frac{2 e}{\nu_{E}(F)}+2 n\right)\left(\Sigma_{s}+\Sigma_{m}^{+}\right) .
$$

Taking into account that by definition $\nu_{E}(F)=\sum_{i=1}^{k} p_{i} \mu_{i} \geq \Sigma_{s}+\Sigma_{m}^{+}$, we get finally:

$$
\sum_{i \in J_{s} \cup J_{m}^{+}} p_{i} m_{i}^{v}<4 n e+4 n^{2}\left(\Sigma_{s}+\Sigma_{m}^{+}\right)
$$

Now the inequalities (10), (20) and (23) imply the following estimate:

$$
\begin{gathered}
\left(4 n^{2}\left(\Sigma_{s}+\Sigma_{m}^{-}\right)+4 n e+4 n^{2}\left(\Sigma_{s}+\Sigma_{m}^{+}\right)\right)\left(\Sigma_{s}+\Sigma_{m}+\Sigma_{u}\right)> \\
>\left(\left(3 \Sigma_{s}+2 \Sigma_{m}+\Sigma_{u}\right) n+e\right)^{2} .
\end{gathered}
$$

Taking into account that $\Sigma_{m}=\Sigma_{m}^{+}+\Sigma_{m}^{-}$, after some easy arithmetic we get the inequality

$$
\left(n\left(\Sigma_{s}-\Sigma_{u}\right)+e\right)^{2}<0
$$

A contradiction.

Q.E.D. for Theorem 4. 


\section{The technique of hypertangent Divisors}

In this section we give the technique of hypertangent divisors and its modification, the technique of hypertangent linear systems. The latter is more flexible and in certain cases more efficient. As a first application, we prove the conditions $(f)$, $(v)$ for regular cyclic covers which in combination with Theorem 2 immediately implies Theorem 1.

2.1. Hypertangent divisors. Consider the following general situation: $X$ is an irreducible projective variety, $x \in X$ a point, and moreover $x$ is either smooth or an isolated singularity. Let $\varphi: X^{+} \rightarrow X$ be the blow up of the point $x, E=\varphi^{-1}(x)$ the exceptional divisor. We assume that $E$ is reduced and irreducible. Let $H$ be an ample divisor on $X$, where, moreover, the linear system $|H|$ is free, that is, defines a finite morphism

$$
\alpha=\alpha_{|H|}: X \rightarrow \mathbb{P}^{\operatorname{dim}|H|}
$$

The degrees of subvarieties $Y \subset X$ are understood in the sense of the divisor $H$, that is, $\operatorname{deg} Y=\left(Y \cdot H^{\operatorname{dim} Y}\right)$, in particular, the degree of the variety $X$ itself is $\operatorname{deg} X=H^{\operatorname{dim} X}=\operatorname{deg} \alpha \cdot \operatorname{deg} \alpha(X)$. The following fact is well known.

Lemma 2.1. For any subvariety $Y \subset X$ the inequality mult $_{y} Y \leq \operatorname{deg} Y$ holds, where $y \in Y$ is an arbitrary point.

Definition 2.1. An effective divisor $D$ on $X$ is said to be hypertangent (with respect to the point $x$ ), if $D^{+} \in|k H-l E|$, where $l \geq k+1, D^{+}$is the strict transform of the divisor $D$ on $X^{+}$. The number $\beta(D)=l / k>1$ is called the slope of the divisor $D$.

Example 2.1. Let $X \subset \mathbb{P}^{N}$ be a hypersurface of degree $d \geq 2, f=q_{1}+q_{2}+$ $\cdots+q_{d}$ its equation with respect to a system of affine coordinates $\left(z_{1}, \ldots, z_{N}\right)$ with the origin at a point $x, q_{i}$ are homogeneous of degree $i$. Let $D_{i}$ be the closure of the set

$$
\left.\left(q_{1}+\cdots+q_{i}\right)\right|_{X}=0
$$

$i \leq d-1$, and assume that $\left.q_{i+1}\right|_{E} \not \equiv 0$. Then $D_{i}$ is a hypertangent divisor with the slope $\frac{i+1}{i}$. On the other hand, if $q_{i+1} \equiv \cdots \equiv q_{k-1} \equiv 0$, but $\left.q_{k}\right|_{E} \not \equiv 0$, then the slope is $\frac{k}{i}$. 
Let $\mathcal{D}$ be a finite set of hypertangent divisors, $\sharp \mathcal{D}=N \leq \operatorname{dim} X-1$. Assume that the equality

$$
\operatorname{codim}_{x}\left(\bigcap_{D \in \mathcal{D}} D\right)=\sharp \mathcal{D}
$$

holds, where $\operatorname{codim}_{x}$ denotes the codimension in a neighborhood of the point $x$.

The following fact is true.

Proposition 2.1 [28,31]. The estimate

$$
\frac{\operatorname{mult}_{x}}{\operatorname{deg}} Y \leq\left(\min _{\mathcal{B} \subset \mathcal{D}}^{*} \prod_{D \in \mathcal{B}} \beta(D)\right)^{-1}
$$

holds, where the symbol min* means that the minimum is taken over all subsets $\mathcal{B} \subset \mathcal{D}$ of cardinality

$$
\sharp \mathcal{B}=\min \{\operatorname{dim} Y-1, N-\operatorname{codim} Y\}=b .
$$

For a detailed proof, see [28]. Here we just remind that the condition (24) implies that there is a subset $\mathcal{B} \subset \mathcal{D}$ of cardinality $b$ such that

$$
\operatorname{codim}_{x}\left(\bigcap_{D \in \mathcal{B}} D \cap Y\right)=\operatorname{codim} Y+b .
$$

Setting $\mathcal{B}=\left\{D_{1}, \ldots, D_{b}\right\}$, we construct a sequence of irreducible subvarieties $Y_{0}=Y, Y_{1}, \ldots, Y_{b}$, where $Y_{i+1}$ is an irreducible component of the effective cycle $\left(Y_{i} \circ D_{i+1}\right)$, satisfying the inequality

$$
\frac{\operatorname{mult}_{x}}{\operatorname{deg}} Y_{i+1} \geq \beta\left(D_{i+1}\right) \frac{\operatorname{mult}_{x}}{\operatorname{deg}} Y_{i}
$$

We complete the proof, applying Lemma 2.1 to the subvariety $Y_{b}$.

2.2. Hypertangent linear systems. In certain cases it is more convenient to work not with hypertangent divisors, but rather with linear systems, generated by those divisors. Similar to Definition 2.1, let us give

Definition 2.2. A non-empty linear system $\Sigma$ on $X$ is said to be hypertangent (with respect to the point $x$ ), if $\Sigma^{+} \subset|k H-l E|$, where $l \geq k+1, \Sigma^{+}$is the strict transform of the system $\Sigma$ on $X^{+}$. The number $\beta(\Sigma)=l / k>1$ is called the slope of the system $\Sigma$. 
The most important characteristic of the system $\Sigma$ is the codimension of its base set in a neighborhood of the point $x, \operatorname{codim}_{x} \operatorname{Bs} \Sigma$.

The set of hypertangent divisors $D \in \mathcal{D}, D^{+} \in\left|k_{D} H-l_{D} E\right|$, generates a hypertangent system $\Sigma_{k}=\Sigma_{k}(\mathcal{D})$ in the following way. Let

$$
f_{D} \in H^{0}\left(X, \mathcal{O}_{X}\left(k_{D} H\right)\right)
$$

be a section, defining the divisor $D$. Set

$$
\Sigma_{k}=\left|\sum_{k_{D} \leq k} f_{D} s_{D}=0\right|,
$$

where the summation is taken over all hypertangent divisors $D \in \mathcal{D}$ such that $k_{D} \leq k$,

$$
s_{D} \in \alpha^{*} H^{0}\left(\mathbb{P}^{\operatorname{dim}|\mathrm{H}|}, \mathcal{O}\left(k-k_{D}\right)\right)
$$

is the pull back of an arbitrary polynomial of degree $\left(k-k_{D}\right)$, with a zero of order $\left(k-k_{D}\right)$ at the point $\alpha(x)$. It is easy to see that

$$
\beta\left(\Sigma_{k}\right) \geq \min _{D \in \mathcal{D}, k_{D} \leq k}\left\{\frac{k+l_{D}-k_{D}}{k}\right\} .
$$

Besides, since $\alpha$ is a finite morphism, we get the equality

$$
\operatorname{codim}_{x} \operatorname{Bs} \Sigma_{k}=\sharp\left\{D \in \mathcal{D} \mid k_{D} \leq k\right\} .
$$

From this equality we see, in particular, that the integer-valued function codim ${ }_{x} \operatorname{Bs}_{k} \Sigma_{k}$ is increasing when $k=k_{D}$ for some $D \in \mathcal{D}$, and for those values of $k$ only. Define the ordering function

$$
\chi:\{1, \ldots, N\} \rightarrow \mathcal{K}=\left\{k_{D} \mid D \in \mathcal{D}\right\}
$$

by the relation

$$
\sharp\left\{D \in \mathcal{D} \mid k_{D}<\chi(i)\right\}<i \leq \sharp\left\{D \in \mathcal{D} \mid k_{D} \leq \chi(i)\right\} .
$$

For instance,

$$
\chi(1)=\min \left\{k_{D} \mid D \in \mathcal{D}\right\}, \quad \chi(N)=\max \left\{k_{D} \mid D \in \mathcal{D}\right\} .
$$

Lemma 2.2. The following inequality holds:

$$
\operatorname{codim}_{x} \operatorname{Bs} \Sigma_{\chi(i)} \geq i \text {. }
$$

Proof: this follows directly from the formula (25) and from the explicit construction of the hypertangent system $\Sigma_{j}$. 
The ordering function is convenient to work with, because for a general set of hypertangent divisors

$$
\mathbb{D}=\left(D_{1}, \ldots, D_{N}\right) \in \prod_{i=1}^{N} \Sigma_{\chi(i)}
$$

and an arbitrary subvariety $Y$ of codimension $l$, containing the point $x$, we get $Y \not \subset$ Supp $D_{i}$ for $i \geq l+1$. In particular, the effective cycle $\left(Y \circ D_{l+1}\right)$ of codimension $l+1$, is well defined and satisfies the inequality

$$
\operatorname{mult}_{x}\left(Y \circ D_{l+1}\right) \geq \beta\left(\Sigma_{l+1}\right) \frac{\operatorname{mult}_{x}}{\operatorname{deg}} Y \operatorname{deg}\left(Y \circ D_{l+1}\right) .
$$

By linearity of this inequality in the cycle $\left(Y \circ D_{l+1}\right)$ there exists an irreducible component of this cycle, an irreducible subvariety $Y_{1}$ of codimension $l+1$, satisfying the inequality

$$
\frac{\operatorname{mult}_{x}}{\operatorname{deg}} Y_{1} \geq \beta\left(\Sigma_{l+1}\right) \frac{\operatorname{mult}_{x}}{\operatorname{deg}} Y_{1} .
$$

Repeating this procedure $b=\min \{\operatorname{dim} Y-1, N-\operatorname{codim} Y\}$ times, we get an irreducible subvariety $Y_{b}$, satisfying the inequality

$$
\frac{\operatorname{mult}_{x}}{\operatorname{deg}} Y_{b} \geq \frac{\operatorname{mult}_{x}}{\operatorname{deg}} Y \prod_{i=1}^{b} \beta\left(\Sigma_{l+i}\right) .
$$

Applying Lemma 2.1 to the subvariety $Y_{b}$ we get

Proposition 2.2. The following estimate holds:

$$
\frac{\operatorname{mult}_{x}}{\operatorname{deg}} Y \leq\left(\prod_{i=1}^{b} \beta\left(\Sigma_{l+i}\right)\right)^{-1} .
$$

The technique of hypertangent divisors and linear systems is applied in most cases to subvarieties of low codimension, mostly to divisors and subvarieties of codimension two, and in the procedure of forming the successive intersections almost all divisors (respectively, linear systems) take part. Moreover, in almost all particular situations for each $D \in \mathcal{D}$ we get $l_{D}=k_{D}+1$, so that all slopes are of the form

$$
\beta(D)=\frac{k_{D}+1}{k_{D}}
$$

and similarly for hypertangent systems. It is easy to see that for those values of the slopes Propositions 2.1 and 2.2 give the same estimates. However, in the most complicated cases one has to argue in a more delicate way, for instance, it is important that the first intersecting hypertangent divisor could be taken 
from the system $\Sigma_{l}$, and not $\Sigma_{l+1}$, in certain cases this can be justified, using some additional considerations, see $[23,26]$. Thus in more complicated cases the technique of hypertangent linear systems is more efficient and flexible than the technique of hypertangent divisors. It is the technique of hypertangent systems that we use below to exclude maximal singularities lying over quadratic points of the fibers. To simplify our exposition, we do not write down explicitly the ordering function (like it was done, for example, in $[29, \S 3]$ ), however, what we mean is exactly the procedure described above.

2.3. Checking the conditions (f) and ( $\mathbf{v})$. As a first application of the technique of hypertangent divisors let us prove that the regular cyclic covers satisfy the condition $(f)$ at any smooth point. First assume that $o \in F$ lies outside the ramification divisor. In the notations of the condition (R1.1) set

$$
D_{i}=\sigma^{*} \overline{\left\{\left.\left(q_{1}+\cdots+q_{i}\right)\right|_{G}=0\right\}},
$$

$i=1, \ldots, m-1$, where the bar means the closure in $\mathbb{P}$, and

$$
L_{j}=\overline{\left\{\left.\left(y-1-\sum_{i=1}^{j} \Phi_{i}\left(w_{1}, \ldots, w_{j}\right)\right)\right|_{F}=0\right\}},
$$

$j=l, l+1, \ldots, K l-1$. Consider first the case $m \leq K l$. In this case set

$$
\mathcal{D}=\left\{D_{i} \mid i=1, \ldots, m-1\right\} \cup\left\{L_{j} \mid j=l, \ldots, K l-2\right\} .
$$

By the regularity condition (R1.1) the codimension of the closed set $\cap_{D \in \mathcal{D}} D$ in a neighborhood of the point $o$ is $\sharp \mathcal{D}=M-1$. Furthermore, by construction

$$
\operatorname{mult}_{o} D_{i}=i+1, \quad \operatorname{mult}_{o} L_{j}=j+1,
$$

so that $D_{i}, L_{j}$ are hypertangent divisors with the slopes $\beta\left(D_{i}\right)=\frac{i+1}{i}$ and $\beta\left(L_{j}\right)=$ $\frac{j+1}{j}$, respectively. Applying Proposition 2.1, we get the estimate

$$
\begin{gathered}
\frac{\operatorname{mult}_{o}}{\operatorname{deg}} Y \leq\left(\frac{1}{2} \cdot \frac{2}{3} \cdot \prod_{i=1}^{m-1} \frac{i+1}{i} \cdot \prod_{j=l}^{K l-2} \frac{j+1}{j}\right)^{-1} \\
\| \\
\frac{3 l}{m(K l-1)}
\end{gathered}
$$


but, since $\frac{3 l}{m(K l-1)}<\frac{4}{m K}$, this proves the condition $(f)$. If $m \geq K l+1$, then set

$$
\mathcal{D}=\left\{D_{i} \mid i=1, \ldots, m-2\right\} \cup\left\{L_{j} \mid j=l, \ldots, K l-1\right\} .
$$

Applying the condition (R1.1) and Proposition 2.1 again, we get the estimate

$$
\begin{gathered}
\frac{\text { mult }_{o}}{\operatorname{deg}} Y \leq\left(\frac{1}{2} \cdot \frac{2}{3} \cdot \prod_{i=1}^{m-2} \frac{i+1}{i} \cdot \prod_{j=l}^{K l-1} \frac{j+1}{j}\right)^{-1} \\
\| \\
\frac{3}{(m-1) K} .
\end{gathered}
$$

Again we get $\frac{3}{(m-1) K}<\frac{4}{m K}$, which proves that the condition $(f)$ holds, if the point $o \in F$ lies outside the branch divisor.

Now assume that the smooth point $o \in F$ lies on the ramification divisor. In this case set

$$
\Delta_{j}=\sigma^{*} \overline{\left\{\left.\left(w_{1}+\cdots+w_{j}\right)\right|_{G}=0\right\}}, \quad j=1, \ldots, K-1,
$$

in the notations of the regularity condition (R2.1).

Lemma 2.3. The inequality mult $_{o} \Delta_{j}=j+1$ holds.

Proof. This is obvious: the polynomial $\left(y^{K}-w_{1}-\cdots-w_{K l}\right)$ vanishes on $F$ and $y(o)=0, j \leq K-1$. Q.E.D. for the lemma.

Now applying Proposition 2.1 to the set of hypertangent divisors

$$
\mathcal{D}=\left\{D_{i} \mid i=1, \ldots, m-1\right\} \cup\left\{\Delta_{j} \mid j=l, \ldots, K-1\right\},
$$

by the condition (R2.1) we get the estimate

$$
\frac{\text { mult }_{o}}{\operatorname{deg}} Y \leq\left(\prod_{i=2}^{m-1} \frac{i+1}{i} \cdot \prod_{j=2}^{K-1} \frac{j+1}{j}\right)^{-1}=\frac{4}{m K},
$$

which is what we need. The condition $(f)$ is shown in full.

Let us prove the condition $(v)$. Assume that for some vertical subvariety $Y$ of codimension two and a smooth point $o \in F_{t} \supset Y$ the inequality (5) does not hold. Let $p=\sigma(o) \in G=\sigma\left(F_{t}\right)$ be the image of the point $o, D=\sigma^{*}\left(T_{p} G \cap G\right)$ the 
tangent divisor. By the regularity conditions mult $_{o} D=2$, so that $Y \neq D$ (and the closed set $D$ is obviously irreducible). Therefore, the effective cycle $Y^{*}=(Y \circ D)$ of codimension two on $F_{t}$ is well defined and satisfies the inequality

$$
\frac{\text { mult }_{o}}{\operatorname{deg}} Y^{*}>\frac{4}{\operatorname{deg} V}
$$

which contradicts the condition $(f)$ which we already proved. This proves the condition $(v)$.

By the sufficient condition of birational superrigidity $[22,25]$ and Theorem 2 this completes the proof of Theorem 1: more precisely, every smooth regular Fano cyclic cover $F$ is birationally superrigid.

\section{ExClusion of maXimal Singularities Lying OVER A DOUble POINT OF}

\section{A FIBER}

In this section, we prove that regular cyclic covers satisfy the condition (ii) of Theorem 4 . The proof breaks naturally into two stages, corresponding to the two cases: when the singular point of the fiber lies outside the ramification divisor and when it lies on this divisor. The connectedness principle of Shokurov and Kollár is used essentially in this proof.

Let $o \in F$ be a singular point of a fiber, $\varphi: F^{+} \rightarrow F$ its blow up, $E=\varphi^{-1}(o) \subset$ $F^{+}$the exceptional divisor, an irreducible quadratic hypersurface in the projective space $\mathbb{P}^{M}$. Assume that a movable linear system $\Sigma \subset\left|-n K_{V}+l F\right|$ has a maximal singularity, the centre of which is the point $o$. From the connectedness principle of Shokurov and Kollár and the inversion of adjunction (which immediately follows from it) [16] we obtain

Proposition 3.1. There exists a hyperplane section $B$ of the quadric E, satisfying the inequality

$$
\operatorname{mult}_{o} D+2 \operatorname{mult}_{B} D^{+}>4 n,
$$

$\left.D \in \Sigma\right|_{F}$ is an arbitrary divisor of the restriction of the linear system $\Sigma$ onto the fiber $F, D^{+}$its strict transform on $F^{+}$.

Proof: see $[32,33]$.

Note that by linearity of the inequality (26) in $D$ one may assume the divisor $D$ to be irreducible. 
3.1. A singular point outside the ramification divisor. In that case we argue following the scheme suggested in $[33, \S 3]$. Assume that $D \in\left|n H_{F}\right|$ satisfies the inequality (26) at the point $o$ for some hyperplane section $B \subset E$, $E=\varphi^{-1}(o) \subset F^{+}$is the exceptional divisor of the blow up $\varphi: F^{+} \rightarrow F$ of the point $o$. Set $p=\sigma(o) \in G, \varphi_{G}: G^{+} \rightarrow G$ to be the blow up of the point $p$, $E_{G}=\varphi_{G}^{-1}(p) \subset G^{+}$to be the exceptional divisor. The morphism $\sigma$ extends to the rational map $\sigma^{+}: F^{+} \rightarrow G^{+}$, and moreover $\sigma^{+}(E)=E_{G}$ and the restriction $\sigma_{E}=\left.\sigma^{+}\right|_{E}: E \rightarrow E_{G}$ is an isomorphism. The blow up $\varphi_{G}$ can be in its turn considered as the restriction onto $G$ of the blow up $\varphi_{\mathbb{P}}: \mathbb{P}^{+} \rightarrow \mathbb{P}$ of the point $p$ in $\mathbb{P}$. Let $E_{\mathbb{P}}=\varphi_{\mathbb{P}}^{-1}(p) \cong \mathbb{P}^{M}$ be the exceptional divisor. Obviously, $E_{G} \subset E_{\mathbb{P}}$ is the smooth quadratic hypersurface, given by the equation $q_{2}=0$.

There is a unique hyperplane $H \subset \mathbb{P}$, containing the point $p$, such that $H^{+} \cap$ $E_{G}=\sigma_{E}(B)$, where $H^{+} \subset \mathbb{P}^{+}$is the strict transform of $H$ on $\mathbb{P}^{+}$. In other words, the projectivized tangent space to $H$ at the point $p$ is that very hyperplane in $E_{\mathbb{P}}$, which cuts out $\sigma_{E}(B)$ on the quadric $E_{G}$. Let $R=\sigma^{-1}(H \cap G) \in\left|H_{F}\right|$ be the corresponding divisor on $F, D_{R} \in\left|n H_{R}\right|$ the restriction of $D$ on $R$. The inequality (26) implies directly the estimate

$$
\operatorname{mult}_{o} D_{R}>4 n \text {. }
$$

By linearity of this inequality in $D_{R}$ we may assume that $D_{R}=Y \subset F$ is an irreducible subvariety of codimension two satisfying the inequality

$$
\frac{\text { mult }_{o}}{\operatorname{deg}} Y>\frac{4}{m K} \text {. }
$$

Now, taking into account the regularity condition (R1.2), we come to a contradiction almost word for word in the same way as in the case of a non-singular point outside the ramification divisor: using the equation $f$, we construct the hypertangent divisors $D_{2}, \ldots, D_{m-1}$, using the equation $g$, we construct the hypertangent divisors $L_{l}, \ldots, L_{K l-1}$ (where $D_{i}, L_{i} \in\left|i H_{F}\right|$ ). By the condition (R1.2)

$$
\operatorname{codim}_{o}\left(\bigcap_{i=2}^{m-1} D_{i}\right) \cap\left(\bigcap_{i=l}^{K l-1} L_{i}\right)=M-1 .
$$

Thus the hypertangent technique (in any of the two versions) gives the estimate

$$
\frac{\text { mult }_{o}}{\operatorname{deg}} Y \leq\left(\prod_{i=4}^{m-1} \frac{i+1}{i}\right)^{-1}\left(\prod_{i=l}^{K l-1} \frac{i+1}{i}\right)^{-1}=\frac{4}{m K}
$$


(we delete from the set $\mathcal{D}=\left\{D_{2}, \ldots, D_{m-1}, L_{l}, \ldots, L_{K l-1}\right\}$ two divisors with the highest slopes $\frac{3}{2}$ and $\frac{4}{3}$ ).

Contradiction.

The case of a maximal singularity lying over a double point of a fiber $o \in F$ outside the ramification divisor is excluded.

3.2. A singular point on the ramification divisor. Let $o \in F$ be a singularity of a fiber lying on the ramification divisor of the morphism $\sigma$. We have the $K$-sheeted cover

$$
F \stackrel{\sigma}{\rightarrow} G \subset \mathbb{P}=\mathbb{P}^{M+1},
$$

$\sigma(o)=p \in G$ is a smooth point of the hypersurface $G, p \in W$. The branch divisor $W_{G}=W \cap G$ has at the point $p$ an isolated quadratic singularity. More precisely (see Sec. 0.4), in some system of affine coordinates $\left(z_{1}, \ldots, z_{M+1}\right)$ on $\mathbb{P}$ with the origin at the point $p$ the hypersurfaces $G$ and $W$ are given by the equations

$$
f=q_{1} \cdots+q_{m}=0, \quad g=w_{1}+\cdots+w_{K l}=0,
$$

where either $q_{1} \equiv w_{1} \equiv z_{M+1}$, or $q_{1} \equiv z_{M+1}$ and $w_{1} \equiv 0$. The germ of the variety $o \in F$ is analytically the germ of the hypersurface $y^{K}=\bar{w}_{2}\left(z_{1}, \ldots, z_{M}\right)+\ldots$, where $\bar{w}_{i}, \bar{q}_{i}$ are the restrictions of the polynomials $w_{i}, q_{i}$ onto the hyperplane $z_{M+1}=0$, in the space $\mathbb{C}_{\left(z_{1}, \ldots, z_{M}, y\right)}^{M+1}$. Let $\varphi: F^{+} \rightarrow F$ be the blow up of the point $o, E \subset F^{+}$the exceptional divisor. It follows from what was said that $E$ realizes naturally as a quadratic hypersurface, $E \subset \mathbb{P}_{\left(z_{1}: \cdots: z_{M}: y\right)}^{M}$. Let $\varphi_{G}: G^{+} \rightarrow G$ be the blow up of the point $p \in G, E_{G}=\varphi_{G}^{-1}(p) \subset G^{+}$the exceptional divisor, $E_{G} \cong \mathbb{P}_{\left(z_{1}: \cdots: z_{M}\right)}^{M-1}$. It is easy to see that the morphism $\sigma$ extends to a rational map $\sigma^{+}: F^{+} \rightarrow G^{+}$, whereas the restriction

$$
\sigma_{E}=\left.\sigma^{+}\right|_{E}: E \rightarrow E_{G}
$$

is the projection of the quadratic cone $E \subset \mathbb{P}^{M}$, given in $\mathbb{P}_{\left(z_{1}: \cdots: z_{M}: y\right)}^{M}$ by the equation $\bar{w}_{2}\left(z_{1}, \ldots, z_{M}\right)=0$, from its vertex $o^{+}=(0: \cdots: 0: 1)$, onto the smooth quadric $E_{+} \subset E_{G}$, given in $E_{G}=\mathbb{P}^{M-1}$ by the very same equation $\bar{w}_{2}=0$. Therefore, $\sigma^{+}$contracts generators of the cone $E$ to points.

By the regularity condition (R2.2), the system of homogeneous equations

$$
\bar{q}_{2}=\cdots=\bar{q}_{m}=\bar{w}_{3}=\cdots=\bar{w}_{K}=0
$$


cuts out on $E_{+}$(and thus on $E$ ) a closed algebraic set, neither component of which is contained in a hyperplane.

Assume that there exist an effective divisor $R \in\left|n H_{F}\right|$ and a hyperplane section $B$ of the quadric cone $E \subset \mathbb{P}^{M}$, satisfying the inequality

$$
\nu+\mu>2 n
$$

where $R^{+} \in\left|n H_{F}-\nu E\right|$, that is, $\nu=\frac{1}{2}$ mult $_{o} R$, and $\mu=\operatorname{mult}_{B} R^{+}, R^{+} \subset F^{+}$is the strict transform of the divisor $R$. By linearity of the inequality (28) one may assume the divisor $R$ to be prime.

Lemma 3.1. The following estimate holds: $\nu \leq \frac{3}{2} n$.

Proof. Assume the converse: $\nu>\frac{3}{2} n$. Let

$$
D_{i}=\sigma^{*}\left(\left.f_{i}\right|_{G}\right), \quad f_{i}=q_{1}+\cdots+q_{i}, \quad i=1, \ldots, m-1,
$$

and

$$
\Delta_{i}=\sigma^{*}\left(\left.g_{i}\right|_{G}\right), \quad g_{i}=w_{1}+\cdots+w_{i}, \quad i=2, \ldots, K-1,
$$

be hypertangent divisors on $F, \mathcal{D}=\left\{D_{1}, \ldots, \Delta_{K-1}\right\}, \sharp \mathcal{D}=m+K-3$. By the regularity condition we get

$$
\operatorname{codim}_{o}\left(\bigcap_{D \in \mathcal{D}} D\right)=\sharp \mathcal{D} .
$$

Lemma 3.2. The prime divisors $D_{1}$ and $R$ are distinct: $D_{1} \neq R$.

Proof. If $\nu \leq 2 n$, then $\mu \geq 1$, that is, $R^{+} \supset B$. By the regularity condition, $D_{1}^{+} \not \supset B$. Therefore, $D_{1}^{+} \neq R^{+}$.

If $\nu>2 n$, then, taking into account that $D_{1}^{+} \in\left|H_{F}-2 E\right|$, we obtain again that $D_{1} \neq R$. Q.E.D. for the lemma.

By the lemma the effective cycle $\left(R \circ D_{1}\right)$ of codimension two is well defined. The inequality

$$
\frac{\text { mult }_{o}}{\operatorname{deg}}\left(R \circ D_{1}\right)>\frac{6}{m K}
$$

holds, so that there is an irreducible subvariety $Y \subset F$ of codimension two (an irreducible component of the cycle $\left.\left(R \circ D_{1}\right)\right)$, satisfying the inequality

$$
\frac{\text { mult }_{o}}{\operatorname{deg}} Y>\frac{6}{m K} \text {. }
$$


Applying to the subvariety $Y$ Proposition 2.1, we obtain the opposite inequality

$$
\frac{\text { mult }_{o}}{\operatorname{deg}} Y \leq \frac{2}{3} \cdots \frac{m-1}{m} \cdot \frac{3}{4} \cdots \frac{K-1}{K}=\frac{6}{m K} .
$$

The contradiction just obtained shows, in addition, that the maximum of the ratio mult $_{o} / \operatorname{deg}$ is attained at the divisor $\sigma^{*} D_{1}$ and equal to $4 / m K$. Q.E.D. for the lemma.

Corollary 3.1. The following inequality holds: $\mu>\frac{1}{2} n$.

3.3. Degrees and multiplicities. Thus the hyperplane section $B$ is really present in the divisor $R^{+} \subset F^{+}$. Now it is more convenient to use the technique of hypertangent linear systems (Sec. 2.2). Set

$$
\Lambda_{i}^{G}=\left|\left(\sum_{j=1}^{i} f_{j} s_{i-j}+\sum_{j=2}^{i} g_{j} r_{i-j}\right)\right|_{G}=0 \mid,
$$

where $s_{k}, r_{k}$ run through the set of all homogeneous polynomials in $z_{1}, \ldots, z_{M+1}$ of degree $k$. Furthermore, let

$$
\Lambda_{i}=\sigma^{*} \Lambda_{i}^{G} \text { and } \Lambda_{i}^{+}
$$

be the pull back of the system $\Lambda_{i}^{G}$ on $F$ and its strict transform on $F^{+}$, respectively. We get $\Lambda_{i}^{+} \subset\left|i H_{F}-(i+1) E\right|$. Finally, let

$$
\Lambda_{i}^{E}=\left.\Lambda_{i}^{+}\right|_{E}
$$

be the projectivized tangent system of the linear system $\Lambda_{i}$ at the point $o$.

Let $H_{E}$ be the class of a hyperplane section of the cone $E$. We get $\Lambda_{i}^{E} \subset$ $\left|(i+1) H_{E}\right|$. In the coordinate form

$$
\Lambda_{i}^{E}=\sigma_{E}^{*}\left|\sum_{j=1}^{i} \bar{q}_{j+1} \bar{s}_{i-j}+\sum_{j=2}^{i} \bar{w}_{j+1} \bar{r}_{i-j}=0\right|,
$$

where $\bar{s}_{k}=\left.s_{k}\right|_{\left\{z_{M+1}=0\right\}}, \bar{r}_{k}=\left.r_{k}\right|_{\left\{z_{M+1}=0\right\}}$. From this explicit presentations and the regularity condition $(\mathrm{R} 2.2)$ we get at once that

$$
\operatorname{codim}_{E} \operatorname{Bs} \Lambda_{i}^{E}=\min \{i, m-1\}+\min \{i, K-1\}-1,
$$

and moreover neither component of the closed set $\operatorname{Bs} \Lambda_{i}^{E}$ is contained in a hyperplane. Note that for $i \geq \max \{m, K\}-1$ we get $\operatorname{Bs} \Lambda_{i}=\operatorname{Bs} \Lambda_{i+1}$ : it is precisely 
the closed set (27). Let

$$
\mathcal{L}=\left(D_{1}, \ldots, D_{m-1}, L_{2}, \ldots, L_{K-1}\right) \in \prod_{i=1}^{m-1} \Lambda_{i} \times \prod_{i=2}^{K-1} \Lambda_{i}
$$

be a general set of hypertangent divisors,

$$
\mathcal{L}^{+}=\left(D_{1}^{+}, \ldots, L_{K-1}^{+}\right) \quad \text { and } \quad \mathcal{L}^{E}=\left(D_{1}^{E}, \ldots, L_{K-1}^{E}\right)=\left.\mathcal{L}^{+}\right|_{E}
$$

its strict transform on $F^{+}$and restriction onto the quadric cone $E$, respectively.

Set $R_{E}=\left.R^{+}\right|_{E}$ to be the projectivized tangent cone of the divisor $R$ at the point $o$. By the regularity condition the closed algebraic set

$$
R_{E} \cap\left(\bigcap_{i=2}^{m-1} D_{i}^{E}\right) \cap\left(\bigcap_{i=2}^{K-1} L_{i}^{E}\right)
$$

is of codimension precisely $m+K-2$ with respect to $E$ (note that in (29) the divisor $D_{1}^{E}$ is omitted).

Therefore, the effective cycle

$$
Y_{E}=\left(R_{E} \circ D_{2}^{E} \circ \cdots \circ D_{m-1}^{E} \circ L_{2}^{E} \circ \cdots \circ L_{K-1}^{E}\right)
$$

of codimension $m+K-2$ on $E$ is well defined. Its $H_{E}$-degree is

$$
\operatorname{deg} Y_{E}=\frac{1}{4} m ! K ! \operatorname{deg} R_{E}=\frac{1}{2} m ! K ! \nu .
$$

Furthermore, by the regularity condition the closed set

$$
R \cap\left(\bigcap_{i=2}^{m-1} D_{i}\right) \cap\left(\bigcap_{i=2}^{K-1} L_{i}\right)
$$

is of codimension $m+K-2$ with respect to $F$ in a neighborhood of the point $o$. Thus we get the uniquely determined effective algebraic cycle $Y$ of codimension $m+K-2$ on $F$, each irreducible component of which contains the point $o$, and which coincides with the effective cycle

$$
\left(R \circ D_{2} \circ \cdots \circ D_{m-1} \circ L_{2} \circ \cdots \circ L_{K-1}\right)_{U}
$$

on a suitable Zariski open set $U \subset F$, containing the point $o$. For a general set $\mathcal{L}$ we get:

$$
Y_{E}=\left(Y^{+} \circ E\right)
$$


is the projectivized tangent cone to $Y$ at the point $o$. However, generally speaking, for the $H_{F}$-degree of the cycle $Y$ we get only the inequality

$$
\operatorname{deg} Y \leq(m-1) !(K-1) ! \operatorname{deg} R=n m ! K !,
$$

since constructing the cycle $Y$, at the intermediate steps (the divisor $R$ is successively intersected with $D_{2}, L_{2}, D_{3}, L_{3}$ and so on) we remove the components that do not contain the point $o$. Note that, generally speaking, certain irreducible components of the cycles $Y$ and $Y_{E}$ can be contained in $D_{1}$ and $D_{1}^{E}$, respectively. Let us separate those components:

$$
Y=Z+Y^{\sharp}, \quad Y_{E}=Z_{E}+Y_{E}^{\sharp},
$$

where Supp $Z \subset D_{1}$ and $Z$ is the maximal subcycle of the effective cycle $Y$ with this property (that is, neither irreducible component of the cycle $Y^{\sharp}$ is contained in $\left.D_{1}\right), Z_{E}=\left(Z^{+} \circ E\right), Y_{E}^{\sharp}=\left(\left(Y^{\sharp}\right)^{+} \circ E\right)=Y_{E}-Z_{E}$. Obviously, Supp $Z_{E} \subset D_{1}^{E}$, but irreducible components of the cycle $Y_{E}^{\sharp}$, generally speaking, can be contained in $D_{1}^{E}$. The following fact is of key importance.

Lemma 3.3. The algebraic cycle

$$
Y_{E}^{\sharp}-\mu\left(B \circ D_{2}^{E} \circ \cdots \circ D_{m-1}^{E} \circ L_{2}^{E} \circ \cdots \circ L_{K-1}^{E}\right)
$$

is effective. In particular, the following inequality holds:

$$
\operatorname{deg} Y_{E}^{\sharp} \geq \frac{1}{2} m ! K ! \mu .
$$

Proof. The first claim holds because by construction the algebraic cycle $R_{E}-$ $\mu B$ is effective, so that the cycle $Y_{E}-\mu B_{\mathcal{L}}$ is effective, either, where $B_{\mathcal{L}}=$ $\left(B \circ D_{2}^{E} \circ \cdots \circ L_{K-1}^{E}\right)$. The support of the cycle $B_{\mathcal{L}}$ is a closed set $\operatorname{Supp} B_{\mathcal{L}}$ of pure codimension $m+K-3$ with respect to $E$. For any irreducible component $\Delta \subset \operatorname{Supp} B_{\mathcal{L}}$ we get $\Delta \not \subset D_{1}^{E}$.

Indeed, assume the converse: $\Delta \subset D_{1}^{E}$. Then $\Delta \subset B$ and

$$
\Delta \subset\left(\bigcap_{i=1}^{m-1} D_{i}^{E}\right) \cap\left(\bigcap_{i=2}^{K-1} L_{i}^{E}\right),
$$

which contradicts the regularity condition (R2.2). 
Therefore, each irreducible component $\Delta$ of the cycle $B_{\mathcal{L}}$ cannot be a component of the cycle $Z_{E}$ and so appears in the cycle $Y_{E}^{\sharp}$ only. This proves the first claim of the lemma. The second claim follows from the first one in a trivial way.

Q.E.D. for the lemma.

Since the irreducble components of the cycle $Y^{\sharp}$ are not contained in the divisor $D_{1}$, the effective cycle $\left(Y^{\sharp} \circ D_{1}\right)$ is well defined, and moreover, the inequality

$$
\frac{\text { mult }_{o}}{\operatorname{deg}}\left(Y^{\sharp} \circ D_{1}\right) \geq 2 \frac{\text { mult }_{o}}{\operatorname{deg}} Y^{\sharp}
$$

holds.

Since for any irreducible subvariety $\Delta \subset F$ we have the estimate $\left(\right.$ mult $\left._{o} / \operatorname{deg}\right) \Delta \leq$ 1 , we get

$$
\operatorname{deg} Y^{\sharp} \geq 2 \operatorname{mult}_{o} Y^{\sharp}=2 \operatorname{deg} Y_{E}^{\sharp} \geq m ! K ! \mu .
$$

On the other hand, we have the inequality

$$
\operatorname{deg} Z \geq \text { mult }_{o} Z=\operatorname{deg} Z_{E}
$$

(which is true for any effective cycle $Z$ ).

Combining these estimates, we get

$$
\begin{gathered}
m ! K ! n \geq \operatorname{deg} Y=\operatorname{deg} Z+\operatorname{deg} Y^{\sharp} \geq \\
\geq \operatorname{deg} Z_{E}+2 \operatorname{deg} Y_{E}^{\sharp}=\operatorname{deg} Y_{E}+\operatorname{deg} Y_{E}^{\sharp} \geq \\
\geq \frac{1}{2} m ! K ! \nu+\frac{1}{2} m ! K ! \mu=\frac{1}{2} m ! K !(\nu+\mu) .
\end{gathered}
$$

Therefore, $\nu+\mu \leq 2 n$. Contradiction.

We have proved that a singular point of a fiber $o \in F$, lying on the ramification divisor of the morphism $\sigma$, cannot be the centre of a maximal singularity of a movable linear system.

This completes our proof of Theorem 3. Q.E.D.

\section{Explicit COnstructions}

In this section we give an explicit construction of varieties with a pencil of Fano cyclic covers. In terms of this construction we check that almost all families of varieties under consideration satisfy the conditions of the criterion of birational 
rigidity. For a few remaining families we construct explicitly non-trivial structures of rationally connected fiber spaces.

4.1. An explicit construction of Fano fiber spaces. Let $a_{*}=\left\{0=a_{0} \leq\right.$ $\left.a_{1} \leq \cdots \leq a_{M+1}\right\}$ be a non-decreasing sequence of non-negative integers, $\mathcal{E}=$ $\bigoplus_{i=0}^{M+1} \mathcal{O}_{\mathbb{P}^{1}}\left(a_{i}\right)$ a locally free sheaf on $\mathbb{P}^{1}, X=\mathbb{P}(\mathcal{E})$ the corresponding projective bundle in the sense of Grothendieck. We have obviously

$$
\operatorname{Pic} X=\mathbb{Z} L_{X} \oplus \mathbb{Z} R, \quad K_{X}=-(M+2) L_{X}+\left(a_{X}-2\right) R,
$$

where $L_{X}$ is the class of the tautological sheaf, $R$ is the class of a fiber of the morphism $\pi_{X}: X \rightarrow \mathbb{P}^{1}, a_{X}=a_{1}+\cdots+a_{M+1}$. Furthermore, it is easy to see that $L_{X}^{M+2}=a_{X}$.

For some $a_{Q}, a_{W} \in \mathbb{Z}_{+}$take divisors

$$
Q \sim m L_{X}+a_{Q} R \quad \text { and } \quad W_{X} \sim K\left(l L_{X}+a_{W} R\right)
$$

on $X$, where $Q \subset X$ is a smooth subvariety, $W=W_{X} \cap Q$ a smooth divisor on $Q$. Let

$$
\sigma: V \rightarrow Q
$$

be the $K$-sheeted cyclic cover of the variety $Q$, branched over the divisor $W$. The projection $\left.\pi_{X}\right|_{Q}$ is denoted by the symbol $\pi_{Q}$, the projection $\pi_{Q} \circ \sigma: V \rightarrow \mathbb{P}^{1}$ by the symbol $\pi$. The fiber $\pi_{Q}^{-1}(t), t \in \mathbb{P}^{1}$, we denote by the symbol $G_{t}$ (or just $G$, when it is clear which point $t \in \mathbb{P}^{1}$ is meant), the fiber $\pi^{-1}(t) \subset V$ by the symbol $F_{t}$ or $F$. Set $L_{Q}=\left.L_{X}\right|_{Q}$ and $L=\sigma^{*} L_{Q}$, respectively. Obviously,

$$
\operatorname{Pic} V=\mathbb{Z} L \oplus \mathbb{Z} F, \quad K_{V}=-L+\left(a_{X}+a_{Q}+(K-1) a_{W}-2\right) F .
$$

It is easy to check the formulas

$$
\left(L^{M} \cdot F\right)=m K, \quad L^{M+1}=K\left(m a_{X}+a_{Q}\right),
$$

whence we get

$$
\left(-K_{V} \cdot L^{M}\right)=K\left((1-m) a_{Q}-m(K-1) a_{W}+2 m\right)
$$

and

$$
\left(K_{V}^{2} \cdot L^{M-1}\right)=K\left(-m a_{X}+(1-2 m) a_{Q}-2 m(K-1) a_{W}+4 m\right) .
$$


It is convenient to use the following notations, introduced in [30]: we write down the parameters of the cover $V$ in the form

$$
\left(\left(a_{1}, \ldots, a_{M+1}\right),\left(a_{Q}, a_{W}\right)\right)
$$

where among the integers $a_{1}, \ldots, a_{M+1}$ we give only non-zero ones, if there are such ones, otherwise we write down simply (0). These notations are convenient because only those covers $V$ require special attention, which have almost all parameters equal to zero. Indeed, the explicit formulas above immediately imply

Proposition 4.1. (i) The variety $V$ satisfies the strong $K^{2}$-condition, that is, the $K^{2}$-condition of depth $0: K_{V}^{2} \notin \operatorname{Int} A_{+}^{2} V$, if one of the following cases takes place:

- $a_{W} \geq 1$,

- $a_{W}=0, a_{Q} \geq 3$,

- $a_{W}=0, a_{Q}=2, a_{X} \geq 1$,

- $a_{W}=0, a_{Q}=1, a_{X} \geq 3$,

- $a_{W}=a_{Q}=0, a_{X} \geq 4$.

(ii) If $a_{W}=0, a_{Q}=2, a_{X}=0$, then the variety $V$ satisfies the $K^{2}$-condition of depth $\frac{2}{m}$.

(iii) If $a_{W}=0, a_{Q}=1$, then the variety $V$ satisfies the $K^{2}$-condition of depth $\frac{1}{m}$ for $a_{X}=2$ and of depth $\left(1+\frac{1}{m}\right)$ for $a_{X}=1$.

(iv) If $a_{W}=a_{Q}=0$, then the variety $V$ satisfies the $K^{2}$-condition of depth 1 for $a_{X}=3$ and of depth 2 for $a_{X}=2$.

Proof: this follows immediately from the inequality $\left(Y \cdot L^{\operatorname{dim} Y}\right) \geq 0$ which is true for any irreducible subvariety $Y$.

Since the $K^{2}$-condition implies the $K$-condition, we get the following list of families that require special attention: 

1. $((0),(0,0))$;
2. $((0),(1,0))$;
3. $((0),(2,0))$;
4. $((1),(0,0))$;
5. $((1),(1,0))$;
6. $((2),(0,0))$;
7. $((1,1),(0,0))$;
8. $((2),(1,0))$;
9. $((1,1),(1,0))$;
10. $((3),(0,0))$;
11. $((1,2),(0,0))$;
12. $((1,1,1),(0,0))$.

In all these cases Proposition 4.1 does not provide the strong $K^{2}$-condition, and in some cases (for instance, for the family 1 ) it is easy to see that the $K^{2}$-condition (of depth 0 ) does not hold. The families $1-12$ will be considered one by one, excluding the family 5 , which will be considered in another paper.

4.2. Varieties of type $((\mathbf{0}),(\mathbf{2}, \mathbf{0}))$. These varieties are similar by their birational properties to the varieties considered in $\left[29\right.$, Sec. 3.1]. Assume that $V / \mathbb{P}^{1}$ is of type $((0),(2,0))$. This means, that $X=\mathbb{P} \times \mathbb{P}^{1}, W=W_{\mathbb{P}} \times \mathbb{P}^{1}$, where $W_{\mathbb{P}} \subset \mathbb{P}$ is a hypersurface of degree $K l$. Let $\sigma_{\mathbb{Y}}: \mathbb{Y} \rightarrow \mathbb{P}$ be the $K$-sheeted cyclic cover, branched over $W_{\mathbb{P}}, \sigma_{Y}: Y \rightarrow X$ the $K$-sheeted cyclic cover, branched over $W$. Obviously, $Y=\mathbb{Y} \times \mathbb{P}^{1}$. Now the variety $V$ identifies naturally with the inverse image $\sigma_{Y}^{-1}(Q) \subset Y$. This construction is valuable because it defines the morphism

$$
p=\left.\operatorname{pr}_{\mathbb{Y}}\right|_{V}: V \rightarrow \mathbb{Y},
$$

where $\operatorname{pr}_{\mathbb{Y}}: Y \rightarrow \mathbb{Y}$ is the projection onto a direct factor.

Lemma 4.1. The degree of the morphism $p$ at a general point equals two. The free anticanonical linear system $\left|-K_{V}\right|=|L|$ defines the morphism $p_{V}=$ $\sigma_{\mathbb{Y}} \circ p: V \rightarrow \mathbb{P}$.

Proof. For a general point $y \in \mathbb{Y}$ the set $p^{-1}(y)$ identifies naturally with the set

$$
Q \cap\left(\left\{\sigma_{\mathbb{Y}}(y)\right\} \times \mathbb{P}^{1}\right)
$$


which consists of two points, since $Q \sim m L_{X}+2 F$, that is, $Q \subset \mathbb{P} \times \mathbb{P}^{1}$ is defined by an equation of bidegree $(2, m)$ :

$$
A\left(x_{*}\right) u^{2}+2 B\left(x_{*}\right) u v+C\left(x_{*}\right) v^{2}=0,
$$

where $A(\cdot), B(\cdot), C(\cdot)$ are homogeneous of degree $m,(u: v)$ and $\left(x_{*}\right)=\left(x_{0}: \cdots\right.$ : $\left.x_{M+1}\right)$ are homogeneous coordinates on $\mathbb{P}^{1}$ and $\mathbb{P}$, respectively. The second claim of the lemma is obvious. See [29, Sec. 3.1] for the details. Q.E.D. for the lemma.

Furthermore, let $\Delta \subset V$ be the subvariety of codimension two, given by the system of equations $A=B=C=0$. The subvariety $\Delta$ is swept out by the lines $L_{y}=\{y\} \times \mathbb{P}^{1}$, which are contracted by the morphism $p$. Set $\Delta_{\mathbb{Y}}=p(\Delta)$. Obviously,

$$
p: V \backslash \Delta \rightarrow \mathbb{Y} \backslash \Delta_{\mathbb{Y}}
$$

is a finite morphism of degree two. Let $\tau \in \operatorname{Bir} V$ be the corresponding Galois involution. It is easy to see that $\tau$ commutes with the generator of the Galois group $\alpha \in$ Aut $V$ of the $K$-sheeted cyclic cover $\sigma: V \rightarrow Q$, so that $\tau$ and $\alpha$ generate a group of $2 K$ elements. Since the involution $\tau$ is biregular outside the invariant closed set $\Delta$ of codimension two, $\tau \in \operatorname{Aut}(V \backslash \Delta)$, the action of $\tau$ on the Picard group Pic $V$ is well defined.

Let $\Sigma \subset\left|-n K_{V}+l F\right|$ be a movable linear system.

Lemma 4.2. (i) The involution $\tau$ transforms the pencil $|F|$ of the fibers of the morphism $\pi$ into the pencil $|m L-F|$. (ii) If $l<0$, then the involution $\tau$ transforms the linear system $\Sigma$ into the linear system

$$
\Sigma^{+} \subset\left|n^{+} L+l^{+} F\right|,
$$

where $n^{+}=n+l m \geq 0, l^{+}=-l>0$.

Proof. Obviously, $\tau^{*} L=L$. Let $F_{t}=\pi^{-1}(t)$ be a fiber. We get

$$
p^{-1}\left(p\left(F_{t}\right)\right)=F_{t} \cup \tau\left(F_{t}\right) \text {. }
$$

But $p\left(F_{t}\right) \sim m \sigma_{\mathbb{Y}}^{*} H_{\mathbb{P}}$, where $H_{\mathbb{P}}$ is the class of a hyperplane section of the projective space $\mathbb{P}$. Since $p^{*} \sigma_{\mathbb{Y}}^{*} H_{\mathbb{Y}}=L$, we obtain the claim (i). Therefore, $\tau^{*} F=$ $m L-F$. This immediately implies the second claim of the lemma.

Now from Lemma 4.2, part (ii) of Proposition 4.1 and Theorem 4 follows

Corollary 4.1. For any movable linear system $\Sigma$ on the variety $V$ its virtual and actual thresholds of canonical adjunction coincide, $c_{\mathrm{virt}}(\Sigma)=c(\Sigma)$, that is, 
the variety $V$ is birationally superrigid. There are exactly two structures of a rationally connected fiber space on $V$ : the pencil $|F|$ of fibers of the projection $\pi$ and its image $\tau_{*}|F|$. The group of birational automorphisms of the variety $V$ is the direct product $\operatorname{Bir} V=(\mathbb{Z} / 2 \mathbb{Z}) \times(\mathbb{Z} / K \mathbb{Z})$, where the factor $\mathbb{Z} / 2 \mathbb{Z}$ is $\{\mathrm{id}, \tau\}$.

4.3. Varieties of type $((\mathbf{1}, \mathbf{1}),(\mathbf{0 , 0}))$. Birational geometry of these varieties is similar to the geometry of double covers of type $((1,1),(0,0))$, considered in [33, Sec. 1.2]. Here $X=\mathbb{P}(\mathcal{E})$, where the sheaf $\mathcal{E}$ is of the form $\mathcal{E}=\mathcal{O}_{\mathbb{P}^{1}}^{\oplus M} \oplus \mathcal{O}_{\mathbb{P}^{1}}(1)^{\oplus 2}$. The space $H^{0}\left(X, \mathcal{L}_{X} \otimes \pi^{*} \mathcal{O}_{\mathbb{P}^{1}}(-1)\right)$ is two-dimensional and determines the pencil of divisors $\left|L_{X}-R\right|$. Its base set $\Delta_{X}=\mathrm{Bs}\left|L_{X}-R\right|$ is of codimension two: it is easy to see that

$$
\Delta_{X}=\mathbb{P}\left(\mathcal{O}_{\mathbb{P}^{1}}^{\oplus M}\right) \cong \mathbb{P}^{M-1} \times \mathbb{P}^{1} .
$$

Furthermore, $Q \sim m L_{X}$ and $W \sim K l L_{X}$. Set

$$
\Delta_{Q}=\Delta_{X} \cap Q, \quad \Delta=\sigma^{-1}\left(\Delta_{Q}\right) \subset V .
$$

Obviously, $\Delta_{Q}$ is a smooth divisor of bidegree $(m, 0)$ on $\Delta_{X}=\mathbb{P}^{M-1} \times \mathbb{P}^{1}, \Delta \subset V$ is a smooth irreducible subvariety of codimension two. We have $-K_{V}=L$, so that the linear system

$$
\left|-K_{V}-F\right|=\sigma^{*}\left(\left.\left|L_{X}-R\right|\right|_{Q}\right)
$$

is movable. Therefore, the variety $V$ does not satisfy the $K$-condition. Let

$$
\varphi: V \rightarrow \mathbb{P}^{1}
$$

be the rational map, given by the pencil $\left|-K_{V}-F\right|$. Birational geometry of the variety $V$ is completely described by

Proposition 4.2. (i) The variety $V$ is birationally superrigid: for any movable linear system $\Sigma$ on $V$ its virtual and actual thresholds of canonical adjunction coincide,

$$
c_{\mathrm{virt}}(\Sigma)=c(\Sigma)
$$

(ii) On the variety $V$ there are exactly two non-trivial structures of a rationally connected fiber space, namely $\pi: V \rightarrow \mathbb{P}^{1}$ and $\varphi: V \rightarrow \mathbb{P}^{1}$. These structures are birationally distinct, that is, there is no birational self-map $\chi \in \operatorname{Bir} V$, transforming the fibers of $\pi$ into the fibers of $\varphi$. The groups of birational and biregular automorphisms of the variety $V$ coincide: $\operatorname{Bir} V=\operatorname{Aut} V$. 
(iii) There exists a unique, up to a fiber-wise isomorphism, Fano fiber space $\pi^{+}: V^{+} \rightarrow \mathbb{P}^{1}$ of the same type $((1,1),(0,0))$, such that the following diagram commutes:

$$
\begin{gathered}
V_{\stackrel{\chi}{\chi} \rightarrow} V^{+} \\
\varphi \downarrow \stackrel{\downarrow}{\downarrow} \quad \pi^{+} \\
\mathbb{P}^{1}=\mathbb{P}^{1},
\end{gathered}
$$

where $\chi$ is a birational map. The correspondence $V \rightarrow V^{+}$is involutive, that is, $\left(V^{+}\right)^{+}=V$.

Proof repeats the proof of Proposition 1.1 in [33] word for word, replacing the double cover by the cyclic cover of degree $K$. Of crucial importance is the birational equivalence

$$
\xi: X \rightarrow \bar{X} \subset \mathbb{P}^{M+3},
$$

given by the complete linear system $\left|L_{X}\right|$, where $\bar{X}$ is the quadric cone with the vertex space $\mathbb{P}^{M-1}=\xi\left(\Delta_{X}\right)$ over a smooth quadric in $\mathbb{P}^{3}$, isomorphic to $\mathbb{P}^{1} \times \mathbb{P}^{1}$. The two pencils of lines on the quadric in $\mathbb{P}^{3}$ determine the two pencils of $(M+1)$-planes on $\bar{X}$. In their turn, these two pencils define the two structures of a rationally connected fiber space on $V$, more precisely, a birational isomorphism $\chi: V \rightarrow V^{+}$, where $V^{+} / \mathbb{P}^{1}$ is a variety of type $((1,1),(0,0))$, and moreover $\chi$ is biregular in codimension one. Now, if $\Sigma \subset\left|-n K_{V}+l F\right|$ is a movable linear system, then either $l \in \mathbb{Z}_{+}$, or for the strict transform

$$
\Sigma^{+}=\chi_{*} \Sigma \subset\left|-n_{+} K_{V^{+}}+l_{+} F^{+}\right|
$$

we get $l_{+} \geq 1$. Obviously, $c(\Sigma)=c\left(\Sigma^{+}\right)$. Since $V$, and thus also $V^{+}$, satisfy the $K^{2}$-condition of depth 2 (Proposition 4.1, (iv)), this implies birational superrigidity. See $[33, \S 1]$ for the details.

\subsection{Varieties with a unique structure of a rationally connected fiber space.}

Theorem 5. A general variety $V$ of any of the following types: $((2),(0,0))$, $((2),(1,0)),((1,1),(1,0)),((3),(0,0)),((1,2),(0,0)),((1,1,1),(0,0))$ is birationally rigid. It has a unique non-trivial structure of a fibration into varieties of negative Kodaira dimension, the projection $\pi: V \rightarrow \mathbb{P}^{1}$. The groups of birational and biregular self-maps of the variety $V$ coincide, $\operatorname{Bir} V=\operatorname{Aut} V$.

Proof. Proposition 4.1, (iii) and (iv) implies that the fiber space $V / \mathbb{P}^{1}$ satisfies the $K^{2}$-condition of depth 2 . In order to apply Theorem 4 , it remains to check the 
$K$-condition for each of the six types, listed in the formulation of the theorem. For varieties of type $((1,1),(1,0))$ it is done word for word the same as in [33, Sec. 1.1]. Let us consider the remaining five types. Here the arguments are similar to [30, Sec. 3.2]. Recall the scheme of the proof.

First let $V / \mathbb{P}^{1}$ be the fiber space of type $((e),(0,0)), e=2$ or 3 . In this case $X=\mathbb{P}(\mathcal{E})$, where

$$
\mathcal{E}=\mathcal{O}_{\mathbb{P}^{1}}(e) \oplus \mathcal{O}_{\mathbb{P}^{1}}^{\oplus(M+1)},
$$

so that the linear system $\left|L_{X}-e R\right|$ consists of the unique divisor $\Delta_{X}=\mathbb{P}^{M} \times \mathbb{P}^{1}$, and the restriction $\left.L_{X}\right|_{\Delta_{X}}$ is the pull back of a hyperplane section of $\mathbb{P}^{M}$ with respect to the projection $\mathbb{P}^{M} \times \mathbb{P}^{1} \rightarrow \mathbb{P}^{M}$. Therefore, $L_{X}$ is trivial on the horizontal lines $\{x\} \times \mathbb{P}^{1}, x \in \mathbb{P}^{M}$. Since $Q \sim m L_{X}$, we get

$$
\Delta_{Q}=Q \cap \Delta_{X}=\Delta_{\mathbb{P}} \times \mathbb{P}^{1},
$$

where $\Delta_{\mathbb{P}} \subset \mathbb{P}^{M}$ is a hypersurface of degree $m$. Obviously, $\Delta=\sigma^{-1}\left(\Delta_{Q}\right) \subset V$ is a divisor of the form $\Delta=\Delta_{V} \times \mathbb{P}^{1}$, where $\sigma_{\Delta}: \Delta_{V} \rightarrow \Delta_{\mathbb{P}}$ is the $K$-sheeted cyclic cover, branched over $\Delta_{W}$, where $W \cap \Delta_{X}=\Delta_{W} \times \mathbb{P}^{1}$. Thus the divisor $\Delta \subset V$ is swept out by the curves of the form $\{y\} \times \mathbb{P}^{1}$, where $y \in \Delta_{V}$. Outside $\Delta_{V} \times \mathbb{P}^{1}$ the morphism $\sigma$ is non-ramified, so that $L$ is trivial on the curves $\{y\} \times \mathbb{P}^{1}, y \in \Delta_{V}$. However, $-K_{V}=L-(e-2) F$, so that the class $\left(-K_{V}\right)$ is non-positive on those curves. Therefore, for any movable system $\Sigma \subset\left|-n K_{V}+l F\right|$ we have $l \in \mathbb{Z}_{+}$, which is what we need (in fact, for $e=3$ our arguments imply that $l \geq n$, but we do not need that). This proves the $K$-condition for the fiber spaces of type $((e),(0,0)), e \in\{2,3\}$.

Let us consider the three remaining families. If $V$ is of type $((2),(1,0))$ or $((1,2),(0,0))$, then on $X$ the linear system $\left|L_{X}-2 R\right|$ is non-empty (it consists of a unique fixed divisor), and so the linear system $|L-2 F|$ is non-empty. If $\Sigma \subset\left|-n K_{V}+l F\right|$ is a movable linear system, then

$$
\left(\left(-n K_{V}+l F\right) \cdot(L-2 F) \cdot L^{M-1}\right) \geq 0 .
$$

For varieties of type $((2),(1,0))$ this gives the estimate

$$
(1-m) n+l m \geq 0,
$$

for varieties of type $((1,2),(0,0))$ the estimate $l \geq 0$. In any case, $l \in \mathbb{Z}_{+}$, that is, the $K$-condition holds. 
Finally, if $V$ is of type $((1,1,1),(0,0))$, then $-K_{V}=L-F$, and moreover, the linear system $\left|-K_{V}\right|$ is movable. Moreover, codim $\operatorname{Bs}|L-F|=3$, so that for the movable linear system $\Sigma \subset\left|-n K_{V}+l F\right|$ we get the estimate

$$
\left(\left(-n K_{V}+l F\right) \cdot(L-F)^{2} \cdot L^{M-2}\right) \geq 0 .
$$

From there we get $l \geq 0$, which is what we need. Proof of Theorem 5 is complete. Q.E.D.

4.5. Non-rigid varieties. On varieties from the remaining three families $((0),(0,0)),((0),(1,0))$ and $((1),(0,0))$ there are infinitely many structures of fibrations into varieties of negative Kodaira dimension, and varieties in these families are not birationally rigid. Let us describe briefly the natural structures of rationally connected fiber spaces on these varieties.

4.5.1. Varieties of type $((0),(0,0))$. Obviously, $V \cong F \times \mathbb{P}^{1}$, where $\sigma: F \rightarrow$ $G$ is a Fano cyclic cover, branched over a divisor $W_{G}=G \cap W^{*}, W^{*} \subset \mathbb{P}$ is a hypersurface of degree $K l$. The projection $q: V \rightarrow F$ onto the direct factor defines on $V$ a structure of a (trivial) conic bundle. Acting by fiber-wise birational automorphisms $\chi \in \operatorname{Bir}(V / F)$ on the pencil $|F|$, we obtain infinitely many pencils of rationally connected varieties on $V$. Let $\tau \in$ Aut $V$ be a generator of the Galois group of the cyclic cover $\sigma: V \rightarrow Q$, so that for general $Q, W$ we get Aut $F=\mathbb{Z} / K \mathbb{Z}=\left\{\right.$ id, $\left.\tau, \ldots, \tau^{K-1}\right\}$.

Conjecture 4.1. For general $G, W_{*}$ there is a unique structure of a conic bundle on $V, q: V \rightarrow F$. For the group of birational automorphisms of the variety $V$ the following presentation holds:

$\operatorname{Bir} V=\operatorname{Aut} F \times \operatorname{Bir}(V / F)=\mathbb{Z} / K \mathbb{Z} \times \operatorname{Bir}(V / F)$.

4.5.2. Varieties of type $((0),(1,0))$. Here $X=\mathbb{P} \times \mathbb{P}^{1}$, the hypersurface $Q \subset X$ is of bidegree $(m, 1)$, the hypersurface $W \subset X$ is of bidegree $(K l, 0)$, that is, $W=W_{\mathbb{P}} \times \mathbb{P}^{1}$, where $W_{\mathbb{P}} \subset P$ is a hypersurface of degree $K l$. Let $(u: v)$ be homogeneous coordinates on $\mathbb{P}^{1},\left(x_{0}: \cdots: x_{M+1}\right)$ homogeneous coordinates on $\mathbb{P}$. The hypersurface $Q$ is given by the equation

$$
u f_{+}+v f_{-}=0
$$


where $f_{ \pm}\left(x_{0}, \ldots, x_{M+1}\right)$ are homogeneous polynomials of degree $m$, the hypersurface $W$ is given by the equation $h\left(x_{*}\right)=0, \operatorname{deg} h=K l$.

Let $\beta_{\mathbb{P}}: Y_{\mathbb{P}} \rightarrow \mathbb{P}$ (respectively, $\beta_{X}: Y_{X} \rightarrow X$ ) be the cyclic cover of the projective space, branched over $W_{\mathbb{P}}$ (respectively, the cyclic cover, branched over $W)$. Obviously, the direct product $X=\mathbb{P} \times \mathbb{P}^{1}$ generates the direct product $Y_{X}=Y_{\mathbb{P}} \times \mathbb{P}^{1}$, compatible with the cyclic covers $\beta_{\mathbb{P}}, \beta_{X}$.

Proposition 4.3. The projection $q: Y_{X} \rightarrow Y_{\mathbb{P}}$ onto the first factor determines a birational morphism

$$
q_{V}=\left.q\right|_{V}: V \rightarrow Y_{\mathbb{P}}
$$

contracting the exceptional divisor $E \subset V$ :

$$
E=q_{V}^{-1}\left(\left\{\beta_{\mathbb{P}}^{*} f_{+}=\beta_{\mathbb{P}}^{*} f_{-}=0\right\}\right) .
$$

The birational morphism $q_{V}$ transforms the pencil $|F|$ of the fibers of the fibration $V / \mathbb{P}^{1}$ into the pencil of divisors

$$
\left\{\lambda_{+} \beta_{\mathbb{P}}^{*} f_{+}+\lambda_{-} \beta_{\mathbb{P}}^{*} f_{-}=0 \mid \lambda_{ \pm} \in \mathbb{C}\right\}
$$

on $Y_{\mathbb{P}}$. The inverse birational map $q_{V}^{-1}$ is the blow up of the base set of this pencil.

Proof: this is obvious.

The variety $Y_{\mathbb{P}}$ is a Fano variety of index $m+1$. Any structure of a fibration $\mathbb{P} \rightarrow S$ into Fano complete intersections of type $\left(b_{1}, \ldots, b_{e}\right)$ with $b_{1}+\cdots+b_{e} \leq$ $m$ generates a structure of a fibration $Y_{\mathbb{P}} \rightarrow S$ into Fano varieties of index $m-b_{1}-\cdots-b_{e}+1 \geq 1$. An example of such a structure is given by the pencil $\left(q_{V}\right)_{*}|F|$ described in Proposition 4.3. Using this method of finding non-trivial structures of a fiber space on $Y_{\mathbb{P}}$ one gets the highest dimension of the base when $b_{1}=\cdots=b_{m}=1$, that is, when $\mathbb{P}$ is fibered into linear subspaces of codimension $m$. In this case $\operatorname{dim} S=m$. The variety $Y_{\mathbb{P}}$ is certainly not birationally rigid.

Conjecture 4.2. If the hypersurface $W_{\mathbb{P}} \subset \mathbb{P}$ is sufficiently general, then for any structure of a rationally connected fiber space $Y_{\mathbb{P}} \rightarrow S$ we have $\operatorname{dim} S \leq m$. If, moreover, $\operatorname{dim} S=m$, then there exist a linear projection $\mathbb{P} \rightarrow \mathbb{P}^{m}$ and a birational map $S \rightarrow \mathbb{P}^{m}$, forming the commutative diagram

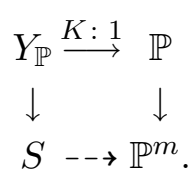


4.5.3. Varieties of type $((\mathbf{1}),(\mathbf{0}, 0))$. It is well known that for $a_{1}=\cdots=$ $a_{M}=0, a_{M+1}=1$ the variety $X=\mathbb{P}(\mathcal{E})$ is isomorphic to the blow up of $\mathbb{P}^{M+2}$ along a subspace of codimension two, and the pencil of fibers of the morphism $\pi_{X}$ corresponds with respect to such an isomorphism to the pencil of hyperplanes in $\mathbb{P}^{M+2}$, passing through the centre of the blow up. From this it is clear that the variety $V$ is birational to a variety $V^{\sharp}$, which is realized as a cyclic $K$-sheeted cover $\sigma^{\sharp}: V^{\sharp} \rightarrow Q^{\sharp} \subset \mathbb{P}^{M+2}$, where $Q^{\sharp}$ is a hypersurface of degree $m$, and $\sigma^{\sharp}$ is ramified (in codimension 1) over the divisor $Q^{\sharp} \cap W^{\sharp}$, where $W^{\sharp} \subset \mathbb{P}^{M+2}$ is a hypersurface of degree $K l$. Thus $V^{\sharp}$ is a Fano variety of index two and any pencil of hyperplanes in $\mathbb{P}^{M+2}$ defines a pencil of Fano varieties on $V^{\sharp}$ and, therefore, a pencil of rationally connected divisors on $V$.

Conjecture 4.3. On the variety $V$ there are no other structures of a rationally connected fiber space, except for the pencils of rationally connected divisors described above. The groups of birational and biregular automorphisms of the variety $V$ coincide:

$$
\operatorname{Bir} V=\text { Aut } V=\mathbb{Z} / K \mathbb{Z} \text {. }
$$

\section{Proof of the Regularity CONDitions}

In this section we prove Theorem 2 on existence of regular cyclic covers: first, we describe the singularities of fibers, then using the methods of [21,25], we prove that a general cyclic cover satisfies the regularity conditions.

5.1. The singularities of fibers. To begin with, let us prove the claim (ii) of Theorem 2. Since the singularity $o \in F$ is isolated and $\operatorname{dim} F>3$, the point $o$ is factorial [4]. Let us describe explicitly its resolution (which also directly implies factoriality). The case, when $o$ lies outside the ramification divisor, is trivial ( $o \in F$ is a non-degenerate quadric singularity). Consider the case when the morphism $\sigma$ is ramified at the point $o$. Here the germ $o \in F$ is analytically a germ of the hypersurface

$$
y^{K}=\bar{w}_{2}\left(z_{1}, \ldots, z_{M}\right)+\ldots
$$

in the space $\mathbb{C}_{\left(z_{1}, \ldots, z_{M}, y\right)}^{M+1}$. Let $\varphi_{1}: \mathbb{C}^{(1)} \rightarrow \mathbb{C}^{M+1}$ be the blow up of the origin, $C^{(1)} \subset \mathbb{C}^{(1)}$ the exceptional divisor, that is, $C^{(1)}=\mathbb{P}_{\left(z_{1}: \cdots: z_{M}: y\right)}^{M}$. Set $\bar{\varphi}: F^{(1)} \rightarrow F$ to be the restriction of $\varphi_{1}$ on $F, E^{(1)}=\bar{\varphi}_{1}^{-1}(o) \subset F^{(1)}$ the exceptional divisor. We have: $E^{(1)} \subset C^{(1)}$ is the quadric $\left\{\bar{w}_{2}=0\right\}$, that is, a quadric cone over a smooth 
quadric in $\mathbb{P}_{\left(z_{1}: \cdots: z_{M}\right)}^{M-1}$ with the vertex at the point $o_{1}=(0: \cdots: 0: 1) \in F^{(1)}$. The germ $o_{1} \in F^{(1)}$ analytically has the form

$$
y^{K-2}=\bar{w}_{2}\left(z_{1}, \ldots, z_{M}\right)+\ldots
$$

If $K=3$, then the point $o_{1} \in F^{(1)}$ (the only potentially singular point of the variety $F^{(1)}$ on the exceptional divisor $\left.E^{(1)}\right)$ is non-singular. If $K=4$, then $o_{1} \in F^{(1)}$ is a non-degenerate quadric singularity, resolved by one blow up. If $K \geq 5$, then we repeat the procedure described above. As a result, we get the sequence of blow ups

$$
\begin{aligned}
& E^{(1)} \quad \ldots \quad E^{([K / 2])} \\
& \cap \cap \cap \\
& F=F^{(0)} \leftarrow F^{(1)} \leftarrow \ldots \leftarrow F^{([K / 2])} \\
& \underset{\cup}{\mathbb{C}^{M+1}}=\underset{\mathbb{C}^{(0)}}{\leftarrow} \underset{\mathbb{C}^{(1)}}{\cap} \underset{\cup}{\stackrel{\cap}{([K / 2])}} \\
& C^{(1)} \quad \ldots \quad C^{([K / 2])}
\end{aligned}
$$

Here $E^{(i)} \subset F^{(i)}$ and $C^{(i)} \subset \mathbb{C}^{(i)}$ are exceptional divisors, $E^{(i)} \subset C^{(i)}$ is a quadric, the rank of which is by one less than the maximum (that is, a cone over a nonsingular quadric in $\left.\mathbb{P}^{M-1}\right), \varphi_{i}: \mathbb{C}^{(i)} \rightarrow \mathbb{C}^{(i-1)}$ blows up the singular point $o_{i-1} \in$ $F^{(i-1)}$, and moreover, $o_{i}$ is the vertex of the cone $E^{(i)}$ for $i=1, \ldots,[K / 2]-1$. If $K \notin 2 \mathbb{Z}$ is odd, then $E^{([K / 2])}$ is also a quadric cone, but the point $o_{[K / 2]} \in F^{([K / 2])}$ is smooth. If $K \in 2 \mathbb{Z}$ is even, then the quadric $E^{([K / 2])}$ is non-degenerate.

Obviously, the canonical class of the variety $F^{([K / 2])}$ is of the form

$$
K_{F}^{([K / 2])}=\varphi^{*} K_{F}+(M-2) \sum_{i-1}^{[K / 2]} E^{(i)},
$$

$\varphi: F^{([K / 2])} \rightarrow F$ is the composition of the blow ups $\varphi_{i}, E^{(i)}$ is understood as the full preimage (pull back) of the divisor $E^{(i)}$ on $F^{([K / 2])}$.

5.2. Existence of regular varieties. First of all, note the following obvious fact: the set of cyclic covers $F$ with at least two singular points or with at least one singularity that does not satisfy the corresponding regularity condition (R1.2) or (R2.2), is a proper closed subset of the set $\mathcal{F}_{\text {sing }}$ and is therefore of codimension $\geq 2$ in $\mathcal{F}$. Therefore, the claim (i) of Theorem 2 is implied by the following fact: 
the set of cyclic covers $F$ with at least one non-regular smooth point is of codimension at least two in $\mathcal{F}$.

In its turn, this claim reduces to a simpler one. Let $p \in \mathbb{P}$ be a fixed point. Consider the set $\mathcal{F}(p)=\{F \in \mathcal{F} \mid G \ni p\} \subset \mathcal{F}$. Since the cover $\sigma$ is cyclic, all the points $o \in \sigma^{-1}(p)$ for $F \in \mathcal{F}(p)$ are either all regular, or all non-regular. Set

$$
\mathcal{F}_{\text {reg }}(p) \subset \mathcal{F}(p)
$$

to be the set of coverings $F$ such that each point $o \in \sigma^{-1}(p)$ is non-singular and regular. We have

Proposition 5.1. The following estimate holds:

$$
\operatorname{codim}_{\mathcal{F}(p)}\left(\mathcal{F}(p) \backslash \mathcal{F}_{\text {reg }}(p)\right) \geq M+2 .
$$

Since $p \in \mathbb{P}^{M+1}$ is an arbitrary point, and $\mathcal{F}(p) \subset \mathcal{F}$ is a divisor, by what was said above Proposition 5.1 implies Theorem 2.

5.3. An estimate for the codimension. Let us prove Proposition 5.1. We work in a fixed system of affine coordinates $z_{1}, \ldots, z_{M+1}$ with the origin at the point $p$, that is, we realize $F$ as a complete intersection of codimension two in $\mathbb{A}_{\left(z_{1}, \ldots, z_{M+1}, y\right)}^{M+2}$. Let us consider separately the two cases, when the conditions (R1.1) and (R2.1) are violated.

Case 1. The point $p$ does not lie on the branch divisor, that is, $w_{0}=1$. We assume that $m \leq K l$ (if $m \geq K l+1$, then the arguments are absolutely similar). Non-regularity of the sequence (3), consisting of $M$ polynomials in $M+1$ variables $z_{*}$, means that for some irreducible cone $B \ni p$ of dimension $\geq 2$ all the polynomials (3) vanish identically on $B$.

Let us construct by induction a sequence of polynomials $\Phi_{i}^{+}\left(w_{1}, \ldots, w_{l}\right)$, setting $\Phi_{l+1}^{+}=\Phi_{l+1}^{\sharp}$ and

$$
\Phi_{i}^{+}=\Phi_{i}^{\sharp}\left(w_{1}, \ldots, w_{l},-K \Phi_{l+1}^{+}, \ldots,-K \Phi_{i-1}^{+}\right) .
$$

Lemma 5.1. The sequence (3) is regular if and only if the sequence

$$
q_{1}, \ldots, q_{m}, w_{l+1}+K \Phi_{l+1}^{+}\left(w_{*}\left(z_{*}\right)\right), \ldots, w_{K l-1}+K \Phi_{K l-1}^{+}\left(w_{*}\left(z_{*}\right)\right)
$$

is regular. 
Proof: this is almost obvious. The sets of common zeros are the same for both sequences (3) and (31). This can be shown by induction using the obvious equality

$$
\left.\left.\Phi_{i}\left(w_{*}\left(z_{*}\right)\right)\right|_{B} \equiv 0 \Leftrightarrow w_{i}\right|_{B} \equiv-\left.K \Phi_{i}^{\sharp}\left(w_{*}\left(z_{*}\right)\right)\right|_{B}
$$

for an arbitrary closed irreducible set B. Q.E.D. for the lemma.

The advantage of the sequence (31) compared to (3) is that the polynomials $w_{i}\left(z_{*}\right)$ are shifted by polynomials $\Phi_{i}^{+}\left(w_{1}\left(z_{*}\right), \ldots, w_{l}\left(z_{*}\right)\right)$, which depend on $w_{1}, \ldots, w_{l}$ only (but not on $w_{l+1}, \ldots, w_{i-1}$ ). The set of polynomials $w_{i}, i \in$ $\{1, \ldots, l\}$ can be assumed to be a fixed set of general position. Thus in (31) each of the polynomials $w_{i}, i \in\{l+1, \ldots, K l-1\}$, is shifted by a fixed homogeneous polynomial of degree $i$.

Let $\Pi$ be the space of polynomials $q_{1}, \ldots, q_{m}, w_{1}, \ldots, w_{K l-1}$. Consider an irreducible component $X \subset \Pi$, corresponding to non-regular sequences (3) or (31). For a fixed set of homogeneous polynomials $u_{1}, \ldots, u_{l}$, $\operatorname{deg} u_{i}=i$, let $\Pi\left(u_{1}, \ldots, u_{l}\right)=\left\{w_{i}=u_{i} \mid i=1, \ldots, l\right\} \subset \Pi$ be the corresponding affine subspace with the fixed $w_{1}, \ldots, w_{l}$, and set $X\left(u_{1}, \ldots, u_{l}\right)=X \cap \Pi\left(u_{1}, \ldots u_{l}\right)$. Obviously, $\Pi\left(u_{1}, \ldots u_{l}\right)$ identifies with the space of polynomials $q_{1}, \ldots, q_{m}, w_{l+1}, \ldots, w_{K l-1}$, which we denote by the symbol $\Pi^{+}$. Thus we consider $X\left(u_{1}, \ldots u_{l}\right)$ to be embedded in the linear space $\Pi^{+}$. For a general set $\left(u_{1}, \ldots u_{l}\right)$ we have

$$
\operatorname{codim}_{\Pi} X=\operatorname{codim}_{\Pi^{+}} X\left(u_{1}, \ldots, u_{l}\right) .
$$

Lemma 5.2. $X(0, \ldots, 0) \neq \emptyset$.

Proof. It is easy to see that the polynomials $\Phi_{i}, \Phi_{i}^{\sharp}$ and $\Phi_{i}^{+}$are quasi-homogeneous in $w_{*}$, where wt $w_{i}=i$. Therefore, for $\lambda \neq 0$

$$
\left(q_{1}, \ldots, q_{m}, \lambda^{l+1} w_{l+1}, \ldots, \lambda^{K l-1} w_{K l-1}\right) \in X\left(\lambda u_{1}, \lambda^{2} u_{2}, \ldots, \lambda^{l} u_{l}\right)
$$

if and only if

$$
\left(q_{1}, \ldots, q_{m}, w_{l+1}, \ldots, w_{K l-1}\right) \in X\left(u_{1}, \ldots, u_{l}\right)
$$

(it is easy to see that the operation $w_{i} \mapsto \lambda^{i} w_{i}$ leaves a non-regular sequence of polynomials in its irreducible component $X$; besides, this operation is invertible). Setting $\lambda=0$, we obtain the claim of the lemma.

Corollary 5.1. $\operatorname{codim}_{\Pi} X \geq \operatorname{codim}_{\Pi^{+}} X(0, \ldots 0)$.

Now it remains to estimate the codimension of the closed set of non-regular sequences $q_{1}, \ldots, q_{m}, w_{l+1}, \ldots, w_{K l-1}$. Let us order these polynomials by increasing 
degrees: $p_{1}=q_{1} \neq 0, p_{2}, \ldots, p_{M}=w_{K l-1}$. Let $Y_{a}, a \geq 2$ be the set of the sets $\left(p_{1}, \ldots, p_{a}\right)$ such that

$$
\operatorname{codim}\left\{p_{1}=\cdots=p_{a}=0\right\}=\operatorname{codim}\left\{p_{1}=\cdots=p_{a-1}=0\right\}=a-1 .
$$

If the sequence $p_{1}, \ldots, p_{M}$ is non-regular, then there is $a \in\{2, \ldots, M\}$, satisfying the equality (32). Therefore, it is sufficient to estimate from below the codimension codim $Y_{a}$ in the space of all sets $\left\{p_{1}, \ldots, p_{a}\right\}$. This can be done using one of the two following simple methods.

Method 1 (used in [21]). Let $\mathbb{P}^{M}=\mathbb{P}\left(\mathbb{C}^{M+1}\right)$ be the projective space with homogeneous coordinates $\left(z_{1}: \cdots: z_{M+1}\right), B \subset \mathbb{P}^{M}$ an irreducible component of the closed set $\left\{p_{1}=\cdots=p_{a-1}=0\right\}$. Let

$$
\gamma: \mathbb{P}^{M} \rightarrow \mathbb{P}^{M+1-a}
$$

be a generic linear projection, $\gamma_{B}: B \rightarrow \mathbb{P}^{M+1-a}$ the corresponding finite morphism, $\gamma_{B}=\left.\gamma\right|_{B}$. For any homogeneous polynomial $h$ on $\mathbb{P}^{M+1-a}$ we have $\gamma_{B}^{*} h \not \equiv 0$, that is, $\left.\gamma^{*} h\right|_{B} \not \equiv 0$. This implies the inequality

$$
\operatorname{codim} Y_{a} \geq\left(\begin{array}{c}
M+1-a+\operatorname{deg} p_{a} \\
M+1-a
\end{array}\right) .
$$

It is easy to check that for $a \in\{2, \ldots, M-1\}$ the right-hand side in (33) is strictly higher than $M+2$, so the estimate (33) is strong enough to prove the inequality (30) provided that regularity is violated at any place except for the last one. If $a=M$, then we have to argue in a different way.

Method 2 (used in $[25,28]$ ). Again let $B \subset \mathbb{P}^{M}$ be an irreducible component of the closed set $\left\{p_{1}=\cdots=p_{M-1}=0\right\}$, which has the correct codimension, that is, $B \subset \mathbb{P}^{M}$ is an irreducible curve. Set $\langle B\rangle$ to be the linear span of the set $B$, codim $<B>\in\{0,1, \ldots, M-1\}$. Take the subset $Y_{M}(b) \subset Y_{M}$, consisting of the sets $\left(p_{1}, \ldots, p_{M}\right)$, such that $\left.p_{M}\right|_{B} \equiv 0$ for some irreducible component $B$ with $\operatorname{codim}<B>=b$.

Lemma 5.3. The following inequality holds:

$$
\operatorname{codim} Y_{M}(b) \geq(M-b)\left(\operatorname{deg} p_{M}+\underset{\substack{I \subset\{1, \ldots, M-1\}, \sharp I=b}}{\min }\left\{\sum_{i \in I} \operatorname{deg} p_{i}\right\}\right)+b+1
$$


Proof: see [25, pp. 72-74].

It is easy to check that for any $b \in\{0, \ldots, M-1\}$ the right-hand side of the inequality (34) is strictly higher than $M+2$. Since

$$
Y_{M}=\bigcup_{b=0}^{M-1} Y_{M}(b),
$$

this completes the proof of Proposition 5.1.

\section{REFERENCES}

[1]. Algebraic surfaces. By the members of the seminar of I.R.Shafarevich. I.R.Shafarevich ed. Proc. Steklov Math. Inst. 75. 1965. English transl. by AMS, 1965. 281 p.

[2]. Artin M., Mumford D. Some elementary examples of unirational varieties which are not rational, Proc. London Math. Soc. V. 25 (1972), 75-95.

[3]. Brown G., Corti A. and Zucconi F. Birational geometry of 3-fold Mori fibre spaces. Proceedings of Fano conference. Torino, Italy. 2004. P. 235-275.

[4]. Call F., Lyubeznik G. A simple proof of Grothendieck's theorem on the parafactoriality of local rings. Contemp. Math. V. 159 (1994), 15-18.

[5]. Clemens H., Griffiths Ph. The intermediate Jacobian of the cubic three-fold. Ann. of Math. V. 95 (1972), 73-100.

[6]. Corti A., Singularities of linear systems and 3-fold birational geometry, in: "Explicit Birational Geometry of Threefolds", London Mathematical Society Lecture Note Series 281 (2000), Cambridge University Press, 259-312.

[7]. Corti A. and Mella M., Birational geometry of terminal quartic 3-folds. I, Amer. J. Math. V. 126 (2004), No. 4, 739-761.

[8]. Corti A., Pukhlikov A. and Reid M., Fano 3-fold hypersurfaces, in: "Explicit Birational Geometry of Threefolds", London Mathematical Society Lecture Note Series 281 (2000), Cambridge University Press, 175-258.

[9]. Graber T., Harris J. and Starr J. Families of rationally connected varieties. J. Amer. Math. Soc. 16 (2002), no. 1, 57-67. 
[10]. Grinenko M.M., Birational automorphisms of a three-dimensional double cone. Sbornik: Mathematics. 189 (1998), no. 7-8, 991-1007.

[11]. Grinenko M.M., Birational properties of pencils of del Pezzo surfaces of degrees 1 and 2. Sbornik: Mathematics. 191 (2000), no. 5-6, 633-653.

[12]. Grinenko M.M., Birational properties of pencils of del Pezzo surfaces of degrees 1 and 2. II. Sbornik: Mathematics. 194 (2003), no. 5-6, 669-696.

[13]. Grinenko M.M., On the double cone over the Veronese surface. Izvestiya: Mathematics. 67 (2003), no. 3, 421-438.

[14]. Griffiths Ph., Harris J. Principles of algebraic geometry. Wiley-Interscience. New York, 1978.

[15]. Iskovskikh V.A. and Manin Yu.I., Three-dimensional quartics and counterexamples to the Lüroth problem, Math. USSR Sb. 86 (1971), no. 1, 140-166.

[16]. Kollár J., et al., Flips and Abundance for Algebraic Threefolds, Asterisque 211, 1993.

[17]. Kollár J., Rational curves on algebraic varieties. Springer-Verlag, Berlin, 1996.

[18]. Kollár J., Miyaoka Y. and Mori S., Rationally connected varieties, J. Alg. Geom. V. 1 (1992), 429-448.

[19]. Pukhlikov A.V., A remark on the theorem of Iskovskikh and Manin on the threedimensional quartic. Proc. Steklov Inst. 208 (1995), 244-254.

[20]. Pukhlikov A.V., Birational automorphisms of three-dimensional algebraic varieties with a pencil of del Pezzo surfaces, Izvestiya: Mathematics 62:1 (1998), 115-155.

[21]. Pukhlikov A.V., Birational automorphisms of Fano hypersurfaces, Invent. Math. 134 (1998), no. 2, 401-426.

[22]. Pukhlikov A.V., Birationally rigid Fano double hypersurfaces, Sbornik: Mathematics 191 (2000), No. 6, 883-908. 
[23]. Pukhlikov A.V., Birationally rigid Fano fibrations, Izvestiya: Mathematics 64 (2000), 131-150.

[24]. Pukhlikov A.V., Essentials of the method of maximal singularities, in "Explicit Birational Geometry of Threefolds", London Mathematical Society Lecture Note Series 281 (2000), Cambridge University Press, 73-100.

[25]. Pukhlikov A.V., Birationally rigid Fano complete intersections, Crelle J. für die reine und angew. Math. 541 (2001), 55-79.

[26]. Pukhlikov A.V., Birationally rigid Fano hypersurfaces with isolated singularities, Sbornik: Mathematics 193 (2002), No. 3, 445-471.

[27]. Pukhlikov A.V., Birationally rigid Fano hypersurfaces, Izvestiya: Mathematics. 66:6 (2002), 1243-1269.

[28]. Pukhlikov A.V., Birationally rigid iterated Fano double covers. Izvestiya: Mathematics. 67 (2003), no. 3, 555-596.

[29]. Pukhlikov A.V., Birationally rigid varieties with a pencil of Fano double covers. I. Sbornik: Mathematics 195 (2004), no. 7, 1039-1071.

[30]. Pukhlikov A.V., Birationally rigid varieties with a pencil of Fano double covers. II. Sbornik: Mathematics 195 (2004), no. 11, 1665-1702.

[31]. Pukhlikov A.V., Birationally rigid Fano varieties, In: Proceedings of Fano conference. Torino, Italy, 2004, 659-681.

[32]. Pukhlikov A.V., Birational geometry of Fano direct products. Izvestiya: Mathematics 69, No. 6, 1225-1255.

[33]. Pukhlikov A.V., Birationally rigid varieties with a pencil of Fano double covers. III, Sbornik: Mathematics 197 (2006), No. 3.

[34]. Pukhlikov A.V., Birational geometry of algebraic varieties with a pencil of Fano complete intersections, arXiv: math.AG/0511090. 
[35]. Sarkisov V.G., Birational automorphisms of conic bundles, Math. USSR Izv. 17 (1981), 177-202.

[36]. Sarkisov V.G., On conic bundle structures, Math. USSR Izv. 20 (1982), no. 2, 354-390.

[37]. Sobolev I. V., On a series of birationally rigid varieties with a pencil of Fano hypersurfaces. Sbornik: Math. 192 (2001), no. 9-10, 1543-1551.

[38]. Sobolev I. V., Birational automorphisms of a class of varieties fibered into cubic surfaces. Izv. Ross. Akad. Nauk Ser. Mat. 66 (2002), no. 1, 203-224.

[39]. Fulton W., Intersection Theory, Springer-Verlag, 1984.

[40]. Cheltsov I.A., On the smooth four-dimensional quintic. Sbornik: Mathematics 191 (2000), no. 9-10, 1399-1419.

[41]. Cheltsov I.A., Non-rationality of a four-dimensional smooth complete intersection of a quadric and a quartic, not containing a plane. Sbornik: Mathematics 194 (2003), no. 11-12, 1679-1699.

[42]. Cheltsov I., Birationally rigid del Pezzo fibrations. Manuscripta Math. 116 (2005), no. $4,385-396$.

[43]. Shokurov V.V., 3-fold log flips, Izvestiya: Mathematics 40 (1993), 93-202.

A.V. Pukhlikov

Department of Mathematical Sciences

M\& O Building, Peach Street

The University of Liverpool

Liverpool L69 7ZL, England

E-mail: pukh@liv.ac.uk,

or

Steklov Institute of Mathematics

Gubkina, 8, 119991 Moscow, Russia

E-mail: pukh@mi.ras.ru 